\title{
Two-scale modeling of fracturing solids using a smeared macro-to-micro discontinuity transition
}

\author{
Erik Svenning $^{1}\left[\right.$ ] Fredrik Larsson ${ }^{1} \cdot$ Martin Fagerström $^{1}$
}

Received: 3 February 2017 / Accepted: 24 May 2017 / Published online: 12 June 2017

(C) The Author(s) 2017. This article is an open access publication

\begin{abstract}
We consider multiscale modeling of fracturing solids undergoing strain localization, whereby Statistical Volume Elements (SVEs) are used to compute the homogenized macroscopic stresses and the eXtended Finite Element Method (XFEM) is used to represent macroscale displacement discontinuities. These discontinuities are imposed on the localized SVEs in a smeared sense, whereby the smearing width is related to the SVE size and the orientation of the macroscopic discontinuity. This smearing width relation, which is derived within the setting of Variationally Consistent Homogenization ( $\mathrm{VCH}$ ), prevents pathological dependence of the solution on the SVE size. The SVE size insensitivity is further improved by adopting the recently proposed localization aligned weakly periodic boundary conditions. Advantages of the proposed method are that it allows multiscale modeling of localized fracture without restrictive assumptions on the SVE size and without the need to explicitly track a localized region in the SVE.
\end{abstract}

Keywords Multiscale modeling - Computational homogenization - Localization - XFEM - Weakly periodic boundary conditions

\section{Introduction}

Multiscale modeling of fracturing solids is a challenging topic that has been the subject of considerable research efforts, cf. the reviews in [1,2]. A frequently used approach

Erik Svenning

erik.svenning@chalmers.se

1 Division of Material and Computational Mechanics, Department of Applied Mechanics, Chalmers University of Technology, Gothenburg, Sweden is to perform numerical simulations on Statistical Volume Elements (SVEs), ${ }^{1}$ whereby a fundamental challenge is that the SVE looses its representative character upon localization. This loss of representativeness leads, for standard first order computational homogenization, to pathological dependence of the numerical results on the macroscale mesh size and the SVE size. These problems have been addressed in the literature using several different schemes. A homogenization scheme that employs discrete cracks on both scales was proposed by Verhoosel et al. [5], where a homogenized traction-separation law is derived, assuming elastic material behavior prior to crack nucleation. An alternative scheme that assumes a weak discontinuity on the microscale was proposed by Coenen et al. [6]. Souza and Allen [7] instead considered visco-elastic materials, and developed a homogenized traction-separation law using interface elements on the microscale and XFEM on the macroscale. Methods employing XFEM were also proposed by Belytschko et al. [8] and Bosco et al. [9], whereas element embedded discontinuities were employed by Toro et al. [10].

The problem with pathological SVE size dependence can be addressed by separating the bulk deformation and the discontinuous deformation in a suitable way, thereby allowing incorporation of a length scale related to the fracture zone. Souza and Allen [7] compute the bulk and interface response from different SVEs, imposing only the discontinuous part of the strain on the localized SVE. Belytschko et al. [8] instead introduce a perforated unit cell, and separate the bulk deformation from the discontinuity by exclud-

\footnotetext{
${ }^{1}$ In the literature, both Representative Volume Element (RVE), Statistical Volume Element (SVE) and Microstructural Volume Element [3] are used to denote a sample of the microstructure. To stress the fact that a sample of finite size will, in general, not be truly representative, we prefer the notion Statistical Volume Element (SVE), cf. Ostoja-Starzewski [4].
} 
ing unstable subdomains. This method allows simulations involving macroscale localization, but requires that the SVE size is approximately equal to the macroscale element size. A scheme that allows wider scale separation was proposed in $[3,6,9]$, where the effective microscale discontinuity is identified by means of image analysis techniques in order to separate the bulk and discontinuity parts of the deformation. In [11], the effective discontinuity was instead identified by monitoring the damage evolution in the SVE. Even though these schemes have successfully addressed several difficulties associated to multiscale localization, we note that previous work tends to focus on approaches where the topology of the microscale discontinuity is explicitly identified and tracked dynamically in different ways [3,6,8-11]. Therefore, a simpler but more versatile approach, that does not require dynamic tracking of the microscale discontinuity, would be very valuable.

Regarding the choice of suitable boundary conditions (BCs) on the SVE, it is well known that both Dirichlet, strong periodic and Neumann BCs are inaccurate if localization occurs in the SVE. In fact, these BCs are inaccurate also during the early stages of crack propagation, i.e. prior to localization, if cracks intersect the SVE boundary, see e.g. the comparison between different BCs in [12]. Remedies proposed in the literature are to mix Dirichlet and Neumann BCs on different parts of the SVE boundary [8], to use Percolation Path Aligned BCs on strong form [3,6], or to use localization aligned periodic BCs on weak form [13].

In the present work, we choose to use localization aligned weakly periodic BCs on the SVE and adopt the concept of Variationally Consistent Homogenization ( $\mathrm{VCH}$ ) to develop a multiscale method that is computationally efficient when the macroscale deformation localizes in a narrow band. We allow for the use of any suitable fracture model (XFEM, interface elements, embedded discontinuities, nonlocal continuum damage) on the microscale, but we consider strong discontinuities on the macroscale, and impose these discontinuities on the SVE in a smeared sense, thereby avoiding the need to explicitly identify and track a localization region in the SVE. More precisely, the homogenized response obtained from the SVEs in the damaged zone provides an (implicit) cohesive zone law, relating the macro discontinuity to the effective macro traction computed from the SVE response. Using a smeared macro-to-micro discontinuity transition to impose the macro discontinuity on the SVE, the macro discontinuity is thereby implicitly driven by the microscale response.

The remainder of the paper is organized as follows: The strong and weak forms of the single scale model problem under consideration are established in Sect. 2, followed by the derivation of the two-scale formulation in Sect. 3, a few illustrative examples in Sect. 4, and some concluding remarks in Sect. 5.

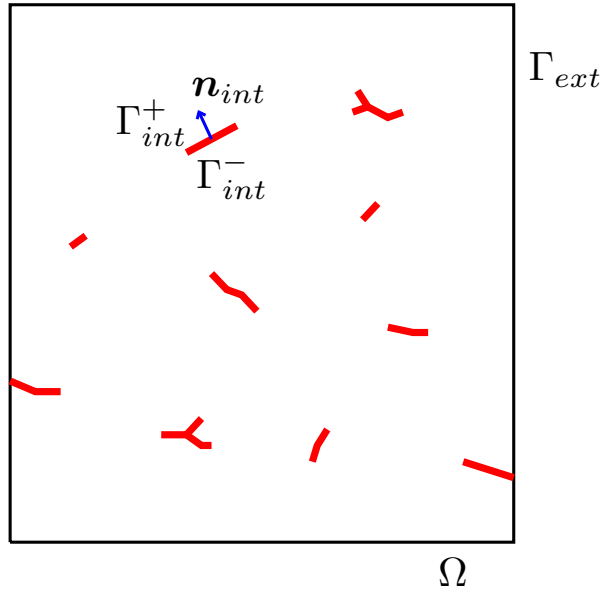

Fig. 1 Specimen with internal boundaries representing cracks in the material

\section{Model problem}

In this section, we will establish the fully resolved problem, i.e., the single scale problem prior to introduction of computational homogenization. To this end, let us consider a solid domain $\Omega$ with external boundary $\Gamma_{\text {ext }}$ and internal surfaces $\Gamma_{i n t}$ representing cracks in the material as indicated in Fig. 1 . The internal boundaries $\Gamma_{i n t}$ have a predefined normal $\boldsymbol{n}_{i n t}$ and consist of two-sided surfaces, as also indicated in Fig. 1. The external boundary consists of a part $\Gamma_{\text {ext }, D}$ with Dirichlet boundary conditions and a part $\Gamma_{\text {ext }, N}$ with Neumann boundary conditions, so that the boundary of $\Omega$ is decomposed as $\partial \Omega=\Gamma_{\text {ext }, D} \cup \Gamma_{\text {ext }, N} \cup \Gamma_{\text {int }}$. Letting superscripts ${ }^{+}$and ${ }^{-}$ denote quantities on the positive side and the negative side of the internal boundaries $\Gamma_{i n t}$, respectively, we define the normal as $\boldsymbol{n}_{\text {int }} \stackrel{\text { def }}{=} \boldsymbol{n}_{\boldsymbol{i n t}}{ }^{-}$. Since the cracks represented by $\Gamma_{\text {int }}$ may branch and intersect, $\boldsymbol{n}_{\text {int }}$ is not necessarily continuous along a chosen part of $\Gamma_{\text {int }}$.

Considering small strains and quasistatic loading, and neglecting the body force, the strong form of the equilibrium equations is given by

$$
\begin{aligned}
-\boldsymbol{\sigma} \cdot \boldsymbol{\nabla} & =\mathbf{0} \text { in } \Omega, \\
\boldsymbol{t}^{+}+\boldsymbol{t}^{-} & =\mathbf{0} \text { on } \Gamma_{\text {int }}, \\
\boldsymbol{t} \stackrel{\text { def }}{=} \boldsymbol{\sigma} \cdot \boldsymbol{n} & =\hat{\boldsymbol{t}} \text { on } \Gamma_{\text {ext }, N}, \\
\boldsymbol{u} & =\hat{\boldsymbol{u}} \text { on } \Gamma_{\text {ext }, D},
\end{aligned}
$$

where $\boldsymbol{\sigma}=\boldsymbol{\sigma}\{\boldsymbol{\epsilon}\}$ is the Cauchy stress, $\boldsymbol{\epsilon}=\left([\boldsymbol{u} \otimes \nabla]^{\text {sym }}\right)$ is the strain, $\boldsymbol{\nabla}$ is the gradient operator, $\boldsymbol{n}$ is a unit normal vector, $\hat{\boldsymbol{t}}$ is a prescribed traction and $\hat{\boldsymbol{u}}$ is a prescribed displacement. The traction on the internal boundaries $\Gamma_{i n t}$ is described by a cohesive zone law specifying $\boldsymbol{t}^{+}=-\boldsymbol{t}^{-}=\boldsymbol{t}\{\llbracket \boldsymbol{u} \rrbracket\}$ in terms of the jump $\llbracket \boldsymbol{u} \rrbracket \stackrel{\text { def }}{=} \boldsymbol{u}^{+}-\boldsymbol{u}^{-}$over the crack faces. Note that in the constitutive relations for the stress-strain relation 
$\sigma=\sigma\{\boldsymbol{\epsilon}\}$ and the traction-separation law $\boldsymbol{t}=\boldsymbol{t}\{\llbracket \boldsymbol{u} \rrbracket\}$, the dependence of $\sigma$ and $\boldsymbol{t}$ on internal variables has been omitted for brevity. Even though body forces have been neglected in Eq. (1), such forces can be included without fundamental difficulty.

The (displacement based, single field) weak solution corresponding to Eq. (1) is obtained by finding $\boldsymbol{u} \in \mathbb{U}$ such that

$$
\begin{gathered}
a(\boldsymbol{u}, \delta \boldsymbol{u})-b(\boldsymbol{t}\{\llbracket \boldsymbol{u} \rrbracket\}, \delta \boldsymbol{u})=l(\delta \boldsymbol{u}) \quad \forall \delta \boldsymbol{u} \in \mathbb{U}^{0}, \\
\mathbb{U}=\left\{\boldsymbol{v}: \boldsymbol{v} \in\left[\mathbb{H}^{1}(\Omega)\right]^{d}, \boldsymbol{v}=\hat{\boldsymbol{u}} \text { on } \Gamma_{\text {ext }, D}\right\}, \\
\mathbb{U}^{0}=\left\{\boldsymbol{v}: \boldsymbol{v} \in\left[\mathbb{H}^{1}(\Omega)\right]^{d}, \boldsymbol{v}=\mathbf{0} \text { on } \Gamma_{\text {ext }, D}\right\},
\end{gathered}
$$

where $d$ is the number of spatial dimensions in the problem, $\boldsymbol{t}\{\llbracket \boldsymbol{u} \rrbracket\}$ denotes the traction obtained from the cohesive zone constitutive law, and where

$$
\begin{aligned}
a(\boldsymbol{u}, \delta \boldsymbol{u}) & \stackrel{\text { def }}{=} \int_{\Omega} \boldsymbol{\sigma}:[\delta \boldsymbol{u} \otimes \nabla] \mathrm{d} \Omega, \\
b(\boldsymbol{t}\{\llbracket \boldsymbol{u} \rrbracket\}, \delta \boldsymbol{u}) & \stackrel{\text { def }}{=} \int_{\Gamma_{\text {int }}} \boldsymbol{t}\{\llbracket \boldsymbol{u} \rrbracket\} \cdot \llbracket \delta \boldsymbol{u} \rrbracket \mathrm{d} \Gamma, \\
l(\delta \boldsymbol{u}) & \stackrel{\text { def }}{=} \int_{\Gamma_{\text {ext }, N}} \hat{\boldsymbol{t}} \cdot \delta \boldsymbol{u} \mathrm{d} \Gamma .
\end{aligned}
$$

Regarding the regularity requirements in $\mathbb{U}$ and $\mathbb{U}^{0}$, $\mathbb{H}^{1}(\Omega)$ denotes the space of square integrable functions with square integrable derivatives, and we recall that $\Omega$ is the "perforated" solid domain.

We remark that Eq. (2) represents the fully resolved problem in the sense that computational homogenization has not yet been introduced. In the next section, we will consider the situation that the fully resolved problem is intractable due to vast separation of length scales and derive a two-scale formulation of this equation.

\section{A two-scale formulation including macroscopic localization}

\subsection{Preliminaries}

In this section, we aim to construct a two-scale model of the problem given by Eq. (2), where $\Gamma_{\text {int }}$ defines a large set of microcracks. On the macroscopic level, we consider the continuous $^{2}$ bulk domain $\bar{\Omega}=\Omega \cup \Gamma_{\text {int }}$, where microscopic cracks are not explicitly resolved. To account for macroscopic strain localization, we split the macroscopic domain according to $\bar{\Omega}=\bar{\Omega}_{r} \cup \bar{\Omega}_{d}$, where $\bar{\Omega}_{r}$ denotes the regular

\footnotetext{
${ }^{2}$ Here, $\bar{\Omega}$ denotes the homogeneous counterpart of $\Omega$, not containing internal boundaries.
}

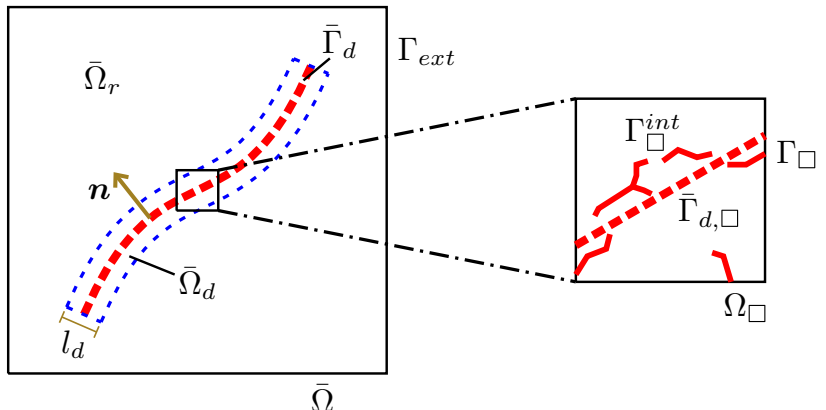

Fig. 2 Macroscopic domain $\bar{\Omega}$ divided into a part $\bar{\Omega}_{d}$ containing an effective macroscopic discontinuity surface $\bar{\Gamma}_{d}$, and a part $\bar{\Omega}_{r}$ that is free from macroscopic discontinuities. The width $l_{d}$ of the discontinuity region $\bar{\Omega}_{d}$ is also shown

part of the continuous bulk domain $\bar{\Omega}$ where the microcracks are separated and the effective (macroscale) response shows no localization. In the discontinuity region $\bar{\Omega}_{d}$, on the other hand, we assume that microcracks have coalesced into macrocracks ${ }^{3}$ causing a macroscale localization. We assume that the localization zone $\bar{\Omega}_{d}$ is narrow, whereby $\bar{\Omega}_{d}$ can be described accurately by the effective discontinuity surface $\bar{\Gamma}_{d}$ and a width $l_{d}$, cf. Fig. 2.

\subsection{Variationally consistent homogenization for continua with macroscopic localization}

To derive a two-scale model, we adopt the procedure of Variationally Consistent Homogenization (VCH), which was introduced by Larsson and Runesson [14] in the context of adaptive scale bridging, and later applied to transient heat flow in [15] and to microfracturing solids in [12]. The key ingredient in the present work, which is an extension of [12], is the introduction of macroscopic localization.

As the first step of $\mathrm{VCH}$, we introduce the decomposition $\mathbb{U}=\mathbb{U}^{\mathrm{M}} \oplus \mathbb{U}^{\mathrm{s}}$ and consider a split of the solution into macro and micro parts according to $\boldsymbol{u}=\boldsymbol{u}^{\mathrm{M}}+\boldsymbol{u}^{\mathrm{s}}$, where $\boldsymbol{u}^{\mathrm{M}} \in \mathbb{U}^{\mathrm{M}}$ describes smooth (average) parts of the solution and $\boldsymbol{u}^{\mathrm{s}} \in \mathbb{U}^{\mathrm{s}}$ are the microscale fluctuations. Assuming the same split for any function in $\mathbb{U}^{0}$, we can state the original problem in Eq. (2) as the macro problem

$a\left(\boldsymbol{u}, \delta \boldsymbol{u}^{\mathrm{M}}\right)-b\left(\boldsymbol{t}\{\llbracket \boldsymbol{u} \rrbracket\}, \delta \boldsymbol{u}^{\mathrm{M}}\right)=l\left(\delta \boldsymbol{u}^{\mathrm{M}}\right) \quad \forall \delta \boldsymbol{u}^{\mathrm{M}} \in \mathbb{U}^{\mathrm{M}, 0}$,

and the micro problem

$a\left(\boldsymbol{u}, \delta \boldsymbol{u}^{\mathrm{s}}\right)-b\left(\boldsymbol{t}\{\llbracket \boldsymbol{u} \rrbracket\}, \delta \boldsymbol{u}^{\mathrm{s}}\right)=l\left(\delta \boldsymbol{u}^{\mathrm{s}}\right) \quad \forall \delta \boldsymbol{u}^{\mathrm{s}} \in \mathbb{U}^{\mathrm{s}, 0}$

\footnotetext{
${ }^{3}$ In fact, any situation where microscale effects (not necessarily microcracks) cause macroscale localization can be considered.
} 
where we recall that $a(\bullet, \bullet), b(\bullet, \bullet)$ and $l(\bullet)$ were defined in Eqs. (3), (4) and (5), respectively. So far, we note that Eqs. (6) and (7) pertain to the Variational MultiScale method (VMS) introduced by Hughes et al. [16].

The second step of $\mathrm{VCH}$ is to restate the integrals in Eq. (6) using running averages in order to obtain a homogenized problem. To this end, we consider the split of the domain $\bar{\Omega}=\bar{\Omega}_{r} \cup \bar{\Omega}_{d}$ as discussed in Sect. 3.1 and, for the regular domain $\bar{\Omega}_{r}$, we introduce the approximation

$$
\begin{gathered}
\int_{\bar{\Omega}_{r}} f \mathrm{~d} \Omega+\int_{\Gamma_{i n t} \cap \bar{\Omega}_{r}} g \mathrm{~d} \Gamma \approx \int_{\bar{\Omega}_{r}} f_{\square} \mathrm{d} \Omega, \\
f_{\square} \stackrel{\text { def }}{=} \frac{1}{\left|\Omega_{\square}\right|}\left[\int_{\Omega_{\square}} f \mathrm{~d} \Omega+\int_{\Gamma_{i n t} \cap \Omega_{\square}} g \mathrm{~d} \Gamma\right],
\end{gathered}
$$

where $\Omega_{\square}$ denotes a Statistical Volume Element (SVE), formally unique for any point in $\bar{\Omega}$.

Remark Note that, if we split the entire domain into adjacent SVEs so that $\bar{\Omega}_{r}=\cup_{i} \Omega_{\square, i}$, and assume the SVE integral $f_{\square}$ to be piecewise constant in each $\Omega_{\square, i}$, the approximation in Eq. (8) becomes an identity.

For the discontinuity region $\bar{\Omega}_{d}$, we state the integrals based on the interface $\bar{\Gamma}_{d}$ according to

$$
\int_{\bar{\Omega}_{d}} f \mathrm{~d} \Omega+\int_{\Gamma_{i n t}^{+} \cap \bar{\Omega}_{d}} g \mathrm{~d} \Gamma \approx \int_{\bar{\Omega}_{d}} f_{\square} \mathrm{d} \Omega \approx \int_{\bar{\Gamma}_{d}} l_{d} f_{\square} \mathrm{d} \Gamma,
$$

where the last approximation is based on the assumption of a narrow region $\bar{\Omega}_{d}$, and where $f_{\square}$, that was defined in Eq. (8), is now evaluated in a point on $\bar{\Gamma}_{d}$.

In the presence of localization, it is clear that the quantity $f_{\square}$ in Eq. (9) will not be representative if evaluated on an arbitrarily sized SVE $\Omega \square$. To overcome this issue, we consider $\bar{\Omega}_{d}$ entirely populated by SVEs through the (narrow) width $l_{d},{ }^{4}$ cf. Fig. 2 . Defining an effective discontinuity surface $\bar{\Gamma}_{d, \square}$ as the plane through the center of the SVE parallel to $\bar{\Gamma}_{d}$, we then obtain the geometric relation

$l_{d}=\frac{\left|\Omega_{\square}\right|}{\left|\bar{\Gamma}_{d, \square}\right|}$.

With this choice, it becomes evident that the homogenization in Eq. (9) is a homogenization on $\bar{\Gamma}_{d}$, whereas the direction perpendicular to $\bar{\Gamma}_{d}$ is fully resolved. We also note that, since $l_{d}$ depends only on the SVE size and the orientation of the effective discontinuity $\bar{\Gamma}_{d}, l_{d}$ is independent of the loading direction.

\footnotetext{
${ }^{4}$ In other words, we choose $l_{d}$ such that it matches the size of $\Omega_{\square}$, whereby the direction perpendicular to $\bar{\Gamma}_{d}$ is fully resolved.
}

Finally, in order to derive the homogenized problem, prolongation conditions need to be specified. More precisely, the macroscale part $\boldsymbol{u}^{\mathrm{M}}$ of the resolved solution inside $\Omega_{\square}$ needs to be expressed in terms of the homogenized solution field $\overline{\boldsymbol{u}}$. To this end, we shall adopt different strategies for $\bar{\Omega}_{r}$ and $\bar{\Omega}_{d}$. We start off with introducing the homogenized displacement ansatz $\overline{\boldsymbol{u}} \in \overline{\mathbb{U}}$ which is smooth on $\hat{\Omega} \stackrel{\text { def }}{=} \bar{\Omega}_{r} \cup \bar{\Omega}_{d} \backslash \bar{\Gamma}_{d}$ but may be discontinuous across $\bar{\Gamma}_{d}$. For SVEs pertaining to the regular subdomain $\bar{\Omega}_{r}$, we use the ansatz from conventional first order homogenization,

$\boldsymbol{u}^{\mathrm{M}}=\left.\boldsymbol{\epsilon}[\overline{\boldsymbol{u}}]\right|_{\overline{\boldsymbol{x}}} \cdot[\boldsymbol{x}-\overline{\boldsymbol{x}}]$ inside $\Omega_{\square}(\overline{\boldsymbol{x}}), \quad \forall \overline{\boldsymbol{x}} \in \bar{\Omega}_{r}$,

where $\Omega_{\square}(\overline{\boldsymbol{x}})$ is the SVE centered around $\overline{\boldsymbol{x}}$.

For SVEs located on $\bar{\Gamma}_{d}$, we adopt a smeared approach, whereby the average strain inside $\Omega_{\square}$ is expressed as the sum of the average bulk strain and the strain contribution from the displacement jump smeared over $l_{d}$. Hence, we state the macroscale displacement field as

$\boldsymbol{u}^{\mathrm{M}}=\left.\overline{\boldsymbol{\epsilon}}_{d}\right|_{\overline{\boldsymbol{x}}} \cdot[\boldsymbol{x}-\overline{\boldsymbol{x}}]$ inside $\Omega_{\square}(\overline{\boldsymbol{x}}), \quad \forall \overline{\boldsymbol{x}} \in \bar{\Gamma}_{d}$

Here, we define the effective strain on $\bar{\Gamma}_{d}$ as

$\overline{\boldsymbol{\epsilon}}_{d} \stackrel{\text { def }}{=} \overline{\boldsymbol{\epsilon}}_{0}+\frac{1}{2 l_{d}}(\llbracket \overline{\boldsymbol{u}} \rrbracket \otimes \boldsymbol{n}+\boldsymbol{n} \otimes \llbracket \overline{\boldsymbol{u}} \rrbracket)$,

where we introduced the bulk strain on $\bar{\Gamma}_{d}$ as ${ }^{5}$

$\overline{\boldsymbol{\epsilon}}_{0} \stackrel{\text { def }}{=} \frac{1}{l_{d}}\left(\int_{s=-l_{d} / 2}^{s=0^{-}} \boldsymbol{\epsilon}[\overline{\boldsymbol{u}}](\overline{\boldsymbol{x}}+s \boldsymbol{n}) \mathrm{d} s\right.$
$\left.+\int_{s=0^{+}}^{s=l_{d} / 2} \boldsymbol{\epsilon}[\overline{\boldsymbol{u}}](\overline{\boldsymbol{x}}+s \boldsymbol{n}) \mathrm{d} s\right)$

and where $\boldsymbol{n}$ is the normal of $\bar{\Gamma}_{d}$ at $\overline{\boldsymbol{x}}$. It is easily verified that the integration of Eq. (12) gives displacement compatibility between $\overline{\boldsymbol{u}}$ and $\boldsymbol{u}^{\mathrm{M}}$ on the boundary of $\bar{\Omega}_{d}$. For the numerical implementation, we further introduce the approximation

$\overline{\boldsymbol{\epsilon}}_{0}[\overline{\boldsymbol{u}}](\overline{\boldsymbol{x}}) \approx \frac{1}{2}\left(\boldsymbol{\epsilon}[\overline{\boldsymbol{u}}]^{+}+\boldsymbol{\epsilon}[\overline{\boldsymbol{u}}]^{-}\right)$,

where the average of the strains

$\boldsymbol{\epsilon}[\overline{\boldsymbol{u}}]^{ \pm} \stackrel{\text { def }}{=} \lim _{s \rightarrow 0} \epsilon[\overline{\boldsymbol{u}}](\overline{\boldsymbol{x}} \pm s \boldsymbol{n})$

replaces the integral expressions.

Using the integral transformations in Eqs. (8) and (9), together with the prolongation expressions in Eqs. (11) and

\footnotetext{
5 Note that the integral expression does not contain the sharp discontinuity $\bar{\Gamma}_{d}$, where $\epsilon[\bar{u}]$ would be unbounded.
} 
(12), we may now restate Eq. (6) as the macroscale problem of finding $\overline{\boldsymbol{u}} \in \overline{\mathbb{U}}$ such that

$$
\begin{gathered}
\int_{\bar{\Omega}_{r}} \overline{\boldsymbol{\sigma}}\{\overline{\boldsymbol{\epsilon}}\}: \delta \overline{\boldsymbol{\epsilon}} \mathrm{d} \Omega+\int_{\bar{\Gamma}_{d}} l_{d} \overline{\boldsymbol{\sigma}}\left\{\overline{\boldsymbol{\epsilon}}_{d}\right\}: \delta \overline{\boldsymbol{\epsilon}}_{d} \mathrm{~d} \Gamma \\
=\int_{\Gamma_{\text {ext }, N}} \hat{\boldsymbol{t}} \cdot \delta \overline{\boldsymbol{u}} \mathrm{d} \Gamma \quad \forall \delta \overline{\boldsymbol{u}} \in \overline{\mathbb{U}}^{0},
\end{gathered}
$$

where $\overline{\boldsymbol{\epsilon}} \stackrel{\text { def }}{=}[\overline{\boldsymbol{u}} \otimes \nabla]^{s y m}$ and the effective macroscale stress $\bar{\sigma}$ is obtained from the microscale solution according to

$\bar{\sigma} \stackrel{\text { def }}{=} \frac{1}{\left|\Omega_{\square}\right|} \int_{\Omega_{\square}} \sigma \mathrm{d} \Omega$.

\subsection{Operational format of integral expressions}

Solving the macroscale problem as given by Eq. (17) requires evaluation of an integral over $\bar{\Omega}_{r}$. This evaluation is inconvenient from a computational perspective, because the finite element mesh will not match $\bar{\Omega}_{r}$. In order to restate $\int_{\bar{\Omega}_{r}} \bullet \mathrm{d} \Omega$, we recall that $\hat{\Omega}=\bar{\Omega}_{r} \cup \bar{\Omega}_{d} \backslash \bar{\Gamma}_{d}$ and use the definition $\int_{\bar{\Omega}_{r}} \bullet \mathrm{d} \Omega=\int_{\hat{\Omega}} \bullet \mathrm{d} \Omega-\int_{\hat{\Omega}_{d}} \bullet \mathrm{d} \Omega$, where $\hat{\Omega}_{d} \stackrel{\text { def }}{=} \bar{\Omega}_{d} \backslash \bar{\Gamma}_{d}$ is the discontinuity region excluding the sharp discontinuity surface. Then, we may employ the approximation

$\int_{\hat{\Omega}_{d}} \overline{\boldsymbol{\sigma}}\{\overline{\boldsymbol{\epsilon}}\}: \delta \overline{\boldsymbol{\epsilon}} \mathrm{d} \Omega \approx \int_{\bar{\Gamma}_{d}} l_{d} \overline{\boldsymbol{\sigma}}\left\{\overline{\boldsymbol{\epsilon}}_{0}\right\}: \delta \overline{\boldsymbol{\epsilon}}_{0} \mathrm{~d} \Gamma$,

where the discontinuity is removed from the argument (i.e. $\overline{\boldsymbol{\epsilon}}_{0}$ and $\left.\delta \overline{\boldsymbol{\epsilon}}_{0}\right)$ to account for the fact that $\hat{\Omega}_{d}$ does not contain $\bar{\Gamma}_{d}$ itself. Finally, we obtain

$$
\begin{aligned}
& \int_{\bar{\Omega}_{r}} \overline{\boldsymbol{\sigma}}\{\overline{\boldsymbol{\epsilon}}\}: \delta \overline{\boldsymbol{\epsilon}} \mathrm{d} \Omega+\int_{\bar{\Gamma}_{d}} l_{d} \overline{\boldsymbol{\sigma}}\left\{\overline{\boldsymbol{\epsilon}}_{d}\right\}: \delta \overline{\boldsymbol{\epsilon}}_{d} \mathrm{~d} \Gamma \approx \\
& \int_{\hat{\Omega}} \overline{\boldsymbol{\sigma}}\{\overline{\boldsymbol{\epsilon}}\}: \delta \overline{\boldsymbol{\epsilon}} \mathrm{d} \Omega+\int_{\bar{\Gamma}_{d}} l_{d}\left(\overline{\boldsymbol{\sigma}}\left\{\overline{\boldsymbol{\epsilon}}_{d}\right\}-\overline{\boldsymbol{\sigma}}\left\{\overline{\boldsymbol{\epsilon}}_{0}\right\}\right): \delta \overline{\boldsymbol{\epsilon}}_{0} \mathrm{~d} \Gamma \\
& \quad+\int_{\bar{\Gamma}_{d}} \overline{\boldsymbol{\sigma}}\left\{\overline{\boldsymbol{\epsilon}}_{d}\right\}:(\llbracket \delta \overline{\boldsymbol{u}} \rrbracket \otimes \boldsymbol{n})^{s y m} \mathrm{~d} \Gamma,
\end{aligned}
$$

where we also used that $\delta \overline{\boldsymbol{\epsilon}}_{d}=\delta \overline{\boldsymbol{\epsilon}}_{0}+\frac{1}{l_{d}}(\llbracket \delta \overline{\boldsymbol{u}} \rrbracket \otimes \boldsymbol{n})^{s y m}$. Inserting the result from Eq. (20) in Eq. (17), the macroscale problem is then to find $\overline{\boldsymbol{u}} \in \overline{\mathbb{U}}$ such that

$$
\bar{a}(\overline{\boldsymbol{u}}, \delta \overline{\boldsymbol{u}})=l(\delta \overline{\boldsymbol{u}}) \quad \forall \delta \overline{\boldsymbol{u}} \in \overline{\mathbb{U}}^{0},
$$

where

$$
\begin{aligned}
\bar{a}(\overline{\boldsymbol{u}}, \delta \overline{\boldsymbol{u}})= & \underbrace{=}_{\text {def }} I_{1} \overline{\int_{\hat{\Omega}}} \overline{\boldsymbol{\sigma}}\{\boldsymbol{\epsilon}[\overline{\boldsymbol{u}}]\}: \boldsymbol{\epsilon}[\delta \overline{\boldsymbol{u}}] \mathrm{d} \Omega \\
& +\underbrace{=}_{\text {def }} I_{2} \\
& +\underbrace{\int_{\bar{\Gamma}_{d}} l_{d}\left(\overline{\boldsymbol{\sigma}}\left\{\overline{\boldsymbol{\epsilon}}_{d}\right\}-\overline{\boldsymbol{\sigma}}\left\{\overline{\boldsymbol{\epsilon}}_{0}[\overline{\boldsymbol{u}}]\right\}\right): \overline{\boldsymbol{\epsilon}}_{0}[\delta \overline{\boldsymbol{u}}] \mathrm{d} \Gamma}_{\stackrel{\text { def }}{=} I_{3}}
\end{aligned}
$$

We note that $I_{1}$ represents the contribution from the effective bulk response, and that $I_{3}$ represents a contribution of cohesive zone type (homogenized from a damaging SVE) since

$\overline{\boldsymbol{\sigma}}\left\{\overline{\boldsymbol{\epsilon}}_{d}\right\}:(\llbracket \delta \overline{\boldsymbol{u}} \rrbracket \otimes \boldsymbol{n})^{s y m}=\left\{\overline{\boldsymbol{t}}_{d} \stackrel{\text { def }}{=}-\overline{\boldsymbol{\sigma}}\left\{\overline{\boldsymbol{\epsilon}}_{d}\right\} \cdot \boldsymbol{n}\right\}=-\overline{\boldsymbol{t}}_{d} \cdot \llbracket \delta \overline{\boldsymbol{u}} \rrbracket$.

Note, however, that the cohesive traction $\overline{\boldsymbol{t}}_{d}$ depends also on the bulk strain because $\overline{\boldsymbol{\sigma}}\left\{\overline{\boldsymbol{\epsilon}}_{d}\right\}=\overline{\boldsymbol{\sigma}}\left\{\overline{\boldsymbol{\epsilon}}_{0}+\frac{1}{l_{d}}[\llbracket \boldsymbol{u} \rrbracket \otimes \boldsymbol{n}]^{\text {sym }}\right\}$. Furthermore, we remark that the novel term $I_{2}$ is scaled by $l_{d}$, and therefore will be negligible for sufficiently small $l_{d}$.

To ensure energy consistency between the micro- and macroscales, the Hill-Mandel condition needs to be fulfilled. Following Larsson et al. [20] it is clear that a Hill-Mandel condition on the form

$$
\begin{aligned}
& \overline{\boldsymbol{\sigma}}\left\{\overline{\boldsymbol{\epsilon}}_{d}\right\}: \delta \overline{\boldsymbol{\epsilon}}_{d} \\
& =\frac{1}{\left|\Omega_{\square}\right|}\left(\int_{\Omega_{\square}} \boldsymbol{\sigma}\{\boldsymbol{\epsilon}\}: \delta \boldsymbol{\epsilon} \mathrm{d} \Omega-\int_{\Gamma_{\square, i n t}} \boldsymbol{t}\{\llbracket \boldsymbol{u} \rrbracket\} \cdot \llbracket \delta \boldsymbol{u} \rrbracket \mathrm{d} \Gamma\right)
\end{aligned}
$$

is met. Utilizing the relation $\overline{\boldsymbol{\epsilon}}_{d}=\overline{\boldsymbol{\epsilon}}_{0}+\frac{1}{l_{d}}(\llbracket \overline{\boldsymbol{u}} \rrbracket \otimes \boldsymbol{n})^{s y m}$, we conclude that

$$
\begin{aligned}
& \overline{\boldsymbol{\sigma}}\left\{\overline{\boldsymbol{\epsilon}}_{d}\right\}: \delta \overline{\boldsymbol{\epsilon}}_{0}-\frac{1}{l_{d}} \overline{\boldsymbol{t}}_{d} \cdot \llbracket \delta \overline{\boldsymbol{u}} \rrbracket \\
& \quad=\frac{1}{\left|\Omega_{\square}\right|}\left(\int_{\Omega_{\square}} \boldsymbol{\sigma}\{\boldsymbol{\epsilon}\}: \delta \boldsymbol{\epsilon} \mathrm{d} \Omega-\int_{\Gamma_{\square, i n t}} \boldsymbol{t}\{\llbracket \boldsymbol{u} \rrbracket\} \cdot \llbracket \delta \boldsymbol{u} \rrbracket \mathrm{d} \Gamma\right),
\end{aligned}
$$

which is the relevant Hill-Mandel condition for an SVE on $\bar{\Gamma}_{d}$.

Regarding the implementation of the macro problem, we choose to use the eXtended Finite Element Method (XFEM) [17-19] for the numerical representation of $\bar{\Gamma}_{d}$. For the numerical solution of Eq. (21), different sets of integration 


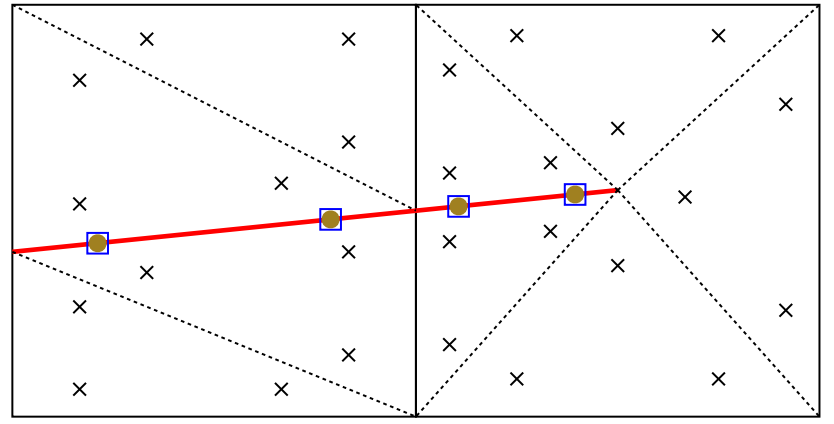

— Macro discontinuity $\bar{\Gamma}_{d}$

........ Subtriangulation

$\times \quad$ Bulk integration point: $\overline{\boldsymbol{\sigma}}\{\overline{\boldsymbol{\epsilon}}\}$

$\square$ Interface integration point: $\overline{\boldsymbol{\sigma}}\left\{\overline{\boldsymbol{\epsilon}_{d}}\right\}$

- Interface integration point: $\overline{\boldsymbol{\sigma}}\left\{\overline{\boldsymbol{\epsilon}_{0}}\right\}$

Fig. 3 Numerical integration of $\bar{a}(\overline{\boldsymbol{u}}, \delta \overline{\boldsymbol{u}})$ in enriched macroscale elements [see Eq. (22)]. $\overline{\boldsymbol{\sigma}}\{\overline{\boldsymbol{\epsilon}}\}$ is computed in bulk integration points to evaluate $I_{1}$. The evaluation of $I_{3}$ requires computation of $\overline{\boldsymbol{\sigma}}\left\{\overline{\boldsymbol{\epsilon}}_{d}\right\}$ in integration points on $\bar{\Gamma}_{d}$, whereas $I_{2}$ requires computation of both $\overline{\boldsymbol{\sigma}}\left\{\overline{\boldsymbol{\epsilon}}_{0}\right\}$ and $\overline{\boldsymbol{\sigma}}\left\{\overline{\boldsymbol{\epsilon}}_{d}\right\}$ on $\bar{\Gamma}_{d}$

points are needed for the evaluation of $\bar{a}(\overline{\boldsymbol{u}}, \delta \overline{\boldsymbol{u}})$ in enriched macroscale elements as shown in Fig. 3. More precisely, the contribution from $I_{1}$ in Eq. (22) is evaluated by computing $\overline{\boldsymbol{\sigma}}\{\overline{\boldsymbol{\epsilon}}\}$ in bulk integration points as shown in the figure. For the contribution from $I_{2}$ and $I_{3}$, we employ two sets of integration points on $\bar{\Gamma}_{d}$ as also shown in the figure. Here, one set of integration points is used to evaluate $\overline{\boldsymbol{\sigma}}\left\{\overline{\boldsymbol{\epsilon}}_{d}\right\}$ and one set of integration points is used to evaluate $\overline{\boldsymbol{\sigma}}\left\{\overline{\boldsymbol{\epsilon}}_{0}\right\}$. We emphasize that both $\overline{\boldsymbol{\sigma}}\{\overline{\boldsymbol{\epsilon}}\}, \overline{\boldsymbol{\sigma}}\left\{\overline{\boldsymbol{\epsilon}}_{d}\right\}$ and $\overline{\boldsymbol{\sigma}}\left\{\overline{\boldsymbol{\epsilon}}_{0}\right\}$ denote the homogenized response of SVEs, where the effective strain imposed on the SVE is $\overline{\boldsymbol{\epsilon}}, \overline{\boldsymbol{\epsilon}}_{d}$ and $\boldsymbol{\epsilon} 0$, respectively.

For small SVE sizes, ${ }^{6}$ i.e. for small $l_{d}$, we may consider the following alternatives when solving Eq. (21):

- Alt. I: Eq. (21) is evaluated as it is, with all terms included:

$$
\begin{aligned}
& \bar{a}(\boldsymbol{u}, \delta \boldsymbol{u})=\int_{\hat{\Omega}} \overline{\boldsymbol{\sigma}}\{\overline{\boldsymbol{\epsilon}}\}: \delta \overline{\boldsymbol{\epsilon}} \mathrm{d} \Omega \\
& +\int_{\bar{\Gamma}_{d}} l_{d}\left(\overline{\boldsymbol{\sigma}}\left\{\overline{\boldsymbol{\epsilon}}_{d}\right\}-\overline{\boldsymbol{\sigma}}\left\{\overline{\boldsymbol{\epsilon}}_{0}\right\}\right): \delta \overline{\boldsymbol{\epsilon}} \mathrm{d} \Gamma \\
& +\int_{\bar{\Gamma}_{d}} \overline{\boldsymbol{\sigma}}\left\{\overline{\boldsymbol{\epsilon}}_{d}\right\}:(\llbracket \delta \overline{\boldsymbol{u}} \rrbracket \otimes \boldsymbol{n})^{s y m} \mathrm{~d} \Gamma, \\
& \overline{\boldsymbol{\sigma}}\left\{\overline{\boldsymbol{\epsilon}}_{d}\right\}=\overline{\boldsymbol{\sigma}}\left\{\overline{\boldsymbol{\epsilon}}_{0}+\frac{1}{l_{d}}(\llbracket \overline{\boldsymbol{u}} \rrbracket \otimes \boldsymbol{n})\right\} .
\end{aligned}
$$

\footnotetext{
${ }^{6}$ With small, we here mean that $l_{\square}$ is small compared to the macroscale dimensions. Note that the SVE size cannot be chosen arbitrarily small: the SVE still needs to be sufficiently large to give a good statistical representation of the microstructure.
}

- Alt. II: $l_{d}$ is assumed to be small, so that $\int_{\bar{\Gamma}_{d}} l_{d}$ $\left(\overline{\boldsymbol{\sigma}}\left\{\overline{\boldsymbol{\epsilon}}_{d}\right\}-\overline{\boldsymbol{\sigma}}\left\{\overline{\boldsymbol{\epsilon}}_{0}\right\}\right): \delta \overline{\boldsymbol{\epsilon}}_{0} \mathrm{~d} \Gamma \approx 0$ :

$$
\begin{aligned}
\bar{a}(\boldsymbol{u}, \delta \boldsymbol{u}) \approx & \int_{\hat{\Omega}} \overline{\boldsymbol{\sigma}}\{\overline{\boldsymbol{\epsilon}}\}: \delta \overline{\boldsymbol{\epsilon}} \mathrm{d} \Omega \\
& +\int_{\bar{\Gamma}_{d}} \overline{\boldsymbol{\sigma}}\left\{\overline{\boldsymbol{\epsilon}}_{d}\right\}:(\llbracket \delta \overline{\boldsymbol{u}} \rrbracket \otimes \boldsymbol{n})^{s y m} \mathrm{~d} \Gamma, \\
\overline{\boldsymbol{\sigma}}\left\{\overline{\boldsymbol{\epsilon}}_{d}\right\}= & \overline{\boldsymbol{\sigma}}\left\{\overline{\boldsymbol{\epsilon}}_{0}+\frac{1}{l_{d}}(\llbracket \overline{\boldsymbol{u}} \rrbracket \otimes \boldsymbol{n})\right\} .
\end{aligned}
$$

- Alt. III: As Alt. II, but the bulk strain in the SVEs on $\bar{\Gamma}_{d}$ is neglected:

$$
\begin{aligned}
\bar{a}(\boldsymbol{u}, \delta \boldsymbol{u}) \approx & \int_{\hat{\Omega}} \overline{\boldsymbol{\sigma}}\{\overline{\boldsymbol{\epsilon}}\}: \delta \overline{\boldsymbol{\epsilon}} \mathrm{d} \Omega \\
& +\int_{\bar{\Gamma}_{d}} \overline{\boldsymbol{\sigma}}\left\{\overline{\boldsymbol{\epsilon}}_{d}\right\}:(\llbracket \delta \overline{\boldsymbol{u}} \rrbracket \otimes \boldsymbol{n})^{s y m} \mathrm{~d} \Gamma, \\
\overline{\boldsymbol{\sigma}}\left\{\overline{\boldsymbol{\epsilon}}_{d}\right\} \approx & \overline{\boldsymbol{\sigma}}\left\{\frac{1}{l_{d}}(\llbracket \overline{\boldsymbol{u}} \rrbracket \otimes \boldsymbol{n})\right\} .
\end{aligned}
$$

We remark that Alt. I and Alt. II require duplicated SVEs in the Gauss points on $\bar{\Gamma}_{d}$, whereas single SVEs are sufficient for Alt. III. Furthermore, we note that Alt. I and Alt. III retain symmetry (if it exists for the original problem), whereas Alt. II is inherently unsymmetric. The performance of these approximations for different SVE sizes will be examined in the numerical examples presented in Sect. 4.

\subsection{Localization aligned weakly periodic boundary conditions}

To solve the microscale problem obtained from Eq. (7) on an SVE $\Omega_{\square}$, the macroscopic part of the displacement given by Eq. (11) or Eq. (12) is imposed on the SVE boundary through suitable BCs. In the present work, we will use weakly periodic boundary conditions that are aligned to the direction of the effective discontinuity in the SVE [13]. To this end, we divide the SVE boundary into an image part $\Gamma_{\square}^{+}$and a mirror part $\Gamma_{\square}^{-}$as shown in Fig. 4a. Furthermore, we introduce a mapping $\varphi_{\text {per }}: \Gamma_{\square}^{+} \rightarrow \Gamma_{\square}^{-}$such that points on $\Gamma_{\square}^{+}$and $\Gamma_{\square}^{-}$ are associated to each other according to $x^{-}=\varphi_{p e r}\left(x^{+}\right)$. For SVEs pertaining to bulk response, i.e. for quadrature points in $\hat{\Omega}$, the mapping is typically performed along horizontal and vertical lines between the surfaces of the square SVE domain. However, for SVEs on the localization band $\bar{\Gamma}_{d}$, the mapping is constructed such that the periodicity directions are aligned with the direction of the effective discontinuity, cf. Fig. 4a. We also define the jump ${ }^{7}$ between a point $\boldsymbol{x}^{+}$on $\Gamma_{\square}^{+}$and the associated point $\boldsymbol{x}^{-}=\varphi_{\text {per }}\left(\boldsymbol{x}^{+}\right)$on

\footnotetext{
$\overline{7}$ Note the difference between the jump $\llbracket \boldsymbol{u} \rrbracket_{\square}$ over the SVE boundaries and the jump $\llbracket \boldsymbol{u} \rrbracket$ over the internal boundaries.
} 


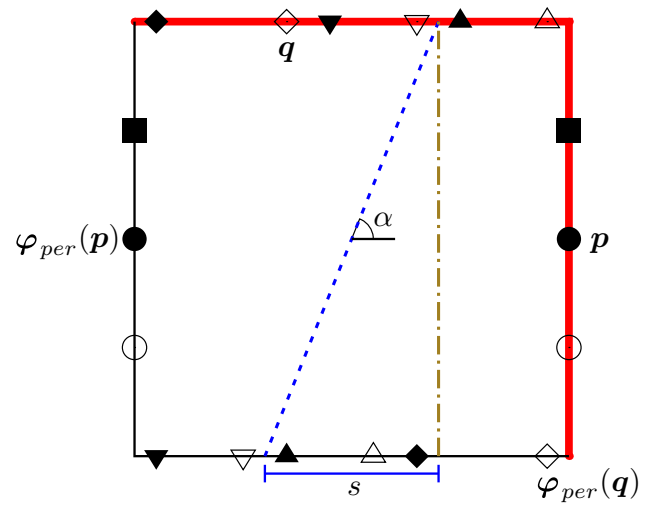

(a)

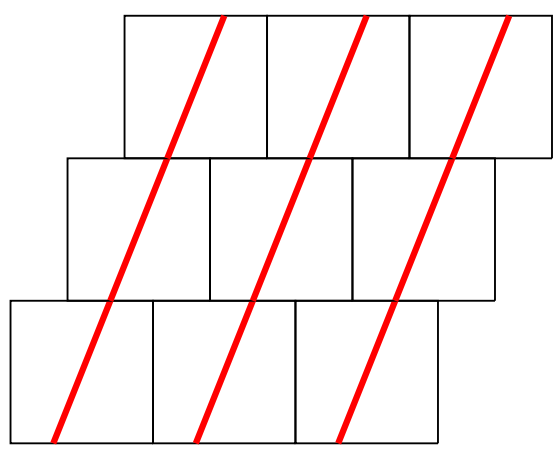

(b)

Fig. 4 Statistical Volume Element (SVE) with boundary divided into image and mirror parts (a). An effective discontinuity passing through the center of the SVE is also shown, together with the shifted mirror function. The corresponding stacking of SVEs is shown in (b)

$\Gamma_{\square}^{-}$as

$\llbracket \boldsymbol{u} \rrbracket_{\square} \stackrel{\text { def }}{=} \boldsymbol{u}_{\Gamma_{\square}^{+}}-\boldsymbol{u}_{\Gamma_{\square}^{-}}=\boldsymbol{u}\left(\boldsymbol{x}^{+}\right)-\boldsymbol{u}\left(\boldsymbol{\varphi}_{\text {per }}\left(\boldsymbol{x}^{+}\right)\right)$on $\Gamma_{\square}^{+}$.

Next, we impose localization aligned weakly periodic boundary conditions on the SVE by introducing an independent discretization for the boundary traction $\boldsymbol{t}_{\lambda}$ and requiring $\llbracket \boldsymbol{u} \rrbracket_{\square}=\overline{\boldsymbol{\epsilon}} \cdot \llbracket \boldsymbol{x}-\overline{\boldsymbol{x}} \rrbracket_{\square}$ to hold in a weak sense on $\Gamma_{\square}^{+}$. The SVE problem is then to find $\boldsymbol{u} \in \mathbb{U}_{\square}$ and $\boldsymbol{t}_{\lambda} \in \mathbb{T}_{\square}$ such that

$$
\begin{aligned}
& a_{\square}(\boldsymbol{u}, \delta \boldsymbol{u})-d_{\square}\left(\boldsymbol{t}_{\lambda}, \delta \boldsymbol{u}\right)=0 \quad \forall \delta \boldsymbol{u} \in \mathbb{U}_{\square}, \\
& \quad-d_{\square}\left(\delta \boldsymbol{t}_{\lambda}, \boldsymbol{u}\right)=-d_{\square}\left(\delta \boldsymbol{t}_{\lambda}, \overline{\boldsymbol{\epsilon}} \cdot[\boldsymbol{x}-\overline{\boldsymbol{x}}]\right) \quad \forall \delta \boldsymbol{t}_{\lambda} \in \mathbb{T}_{\square}, \\
& \mathbb{U}_{\square}=\left\{\boldsymbol{v}: \boldsymbol{v} \in\left[\mathbb{H}^{1}\left(\Omega_{\square}\right)\right]^{d}, \int_{\Gamma_{\square}} \boldsymbol{v} \mathrm{d} \Gamma=\mathbf{0}\right\}, \\
& \mathbb{T}_{\square}=\left\{\boldsymbol{t}: \boldsymbol{t} \in\left[\mathbb{L}_{2}\left(\Gamma_{\square}^{+}\right)\right]^{d}\right\},
\end{aligned}
$$

where we introduced the expressions

$$
\begin{aligned}
& a_{\square}(\boldsymbol{u}, \delta \boldsymbol{u}) \stackrel{\text { def }}{=} \frac{1}{\left|\Omega_{\square}\right|}\left[\int_{\Omega_{\square}} \boldsymbol{\sigma}: \boldsymbol{\epsilon}[\delta \boldsymbol{u}] \mathrm{d} \Omega\right. \\
& \left.-\int_{\Gamma_{\square, \text { int }}} \boldsymbol{t} \cdot \llbracket \delta \boldsymbol{u} \rrbracket \mathrm{d} \Gamma\right], \\
& d_{\square}\left(\boldsymbol{t}_{\lambda}, \delta \boldsymbol{u}\right) \stackrel{\text { def }}{=} \frac{1}{\left|\Omega_{\square}\right|} \int_{\Gamma_{\square}^{+}} \boldsymbol{t}_{\lambda} \cdot \llbracket \delta \boldsymbol{u} \rrbracket_{\square} \mathrm{d} \Gamma,
\end{aligned}
$$

and where $\mathbb{L}_{2}\left(\Gamma_{\square}^{+}\right)$denotes the space of square integrable functions on $\Gamma_{\square}^{+}$. See $[13,20,21]$ for further details.

We remark that, on $\bar{\Gamma}_{d}$, the weakly periodic boundary conditions are employed to impose the macroscopic strain $\overline{\boldsymbol{\epsilon}}_{d}=\overline{\boldsymbol{\epsilon}}_{0}+\frac{1}{l_{d}}(\llbracket \boldsymbol{u} \rrbracket \otimes \boldsymbol{n})^{s y m}$ in a weak sense on the whole SVE boundary. Hence, there is no need to explicitly identify the location or width of the damaged zone in the SVE, only the localization direction needs to be determined in order to define the effective discontinuity normal direction $\boldsymbol{n}$. Recall that $\boldsymbol{n}$ also defines the periodicity alignment.

We also remark that $l_{d}$ cannot be chosen freely, it is related to the SVE size $\left|\Omega_{\square}\right|$ according to Eq. (10). For the special case of $2 \mathrm{D}$ and a square SVE, we may compute $l_{d}$ explicitly as

$$
\begin{array}{ll}
l_{d}=l_{\square} \cos \alpha, & 0^{\circ} \leq \alpha \leq 45^{\circ}, \\
l_{d}=l_{\square \sin \alpha,} & 45^{\circ} \leq \alpha \leq 90^{\circ},
\end{array}
$$

where $l_{\square}=\sqrt{\left|\Omega_{\square}\right|}$ and $\alpha$ is the angle between the effective discontinuity and the $x$-axis.

To summarize, we may compute the homogenized stress $\overline{\boldsymbol{\sigma}}=\overline{\boldsymbol{\sigma}}\{\overline{\boldsymbol{\epsilon}}\}$ for a given $\overline{\boldsymbol{\epsilon}}$ by solving Eq. (32) and employing Eq. (18).

\section{Numerical examples}

\subsection{Preliminaries}

In this section, we demonstrate the performance of the proposed method with a few numerical examples. To assess the accuracy of the proposed method, we will present comparisons between fully resolved Direct Numerical Simulations (DNS) and two-scale simulations $\left(\mathrm{FE}^{2}\right)$. The open source software package OOFEM [22,23] has been used for the numerical implementation.

In Example 4.2, we present a comparison between Alt. I, Alt. II and Alt. III described in Sect. 3. In Examples 4.3 and 4.4, we use Alt. I and demonstrate that the proposed method accurately predicts homogenized stress-strain relations, provided that an appropriate kinematic ansatz is made for the macroscale displacement field. Finally, an outlook is 
Fig. 5 Specimen with vertical localization band (dashed line) considered in Example 4.2

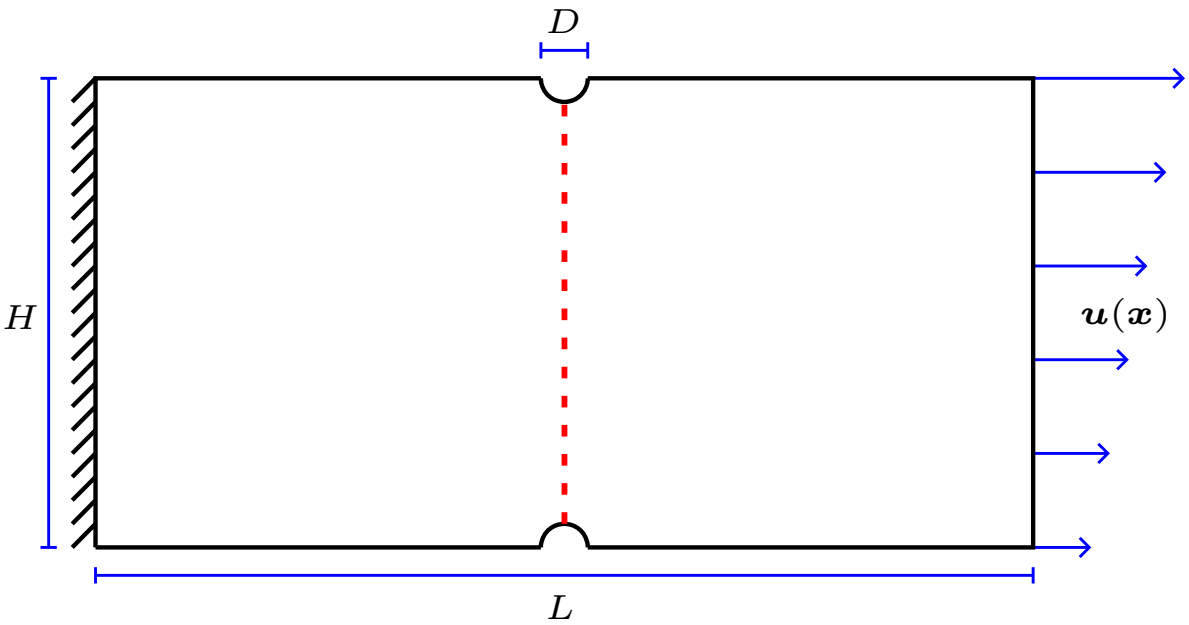

Fig. 6 Deformed shape of the specimen considered in Example 4.2, magnified by a factor of 100 . The figure is colored by the horizontal displacement. (Color figure online)

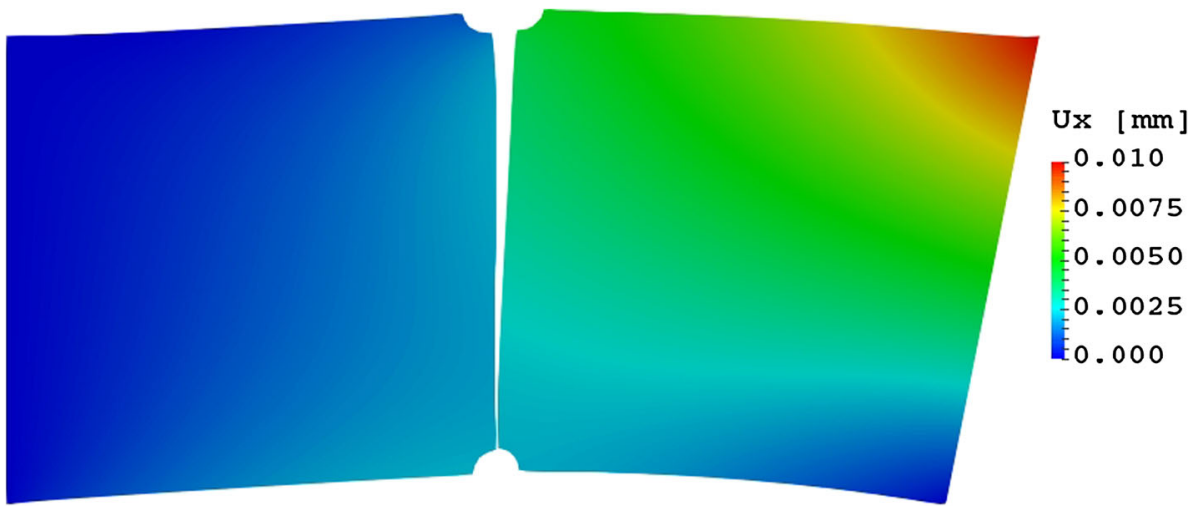

presented in Example 4.5, indicating the application of the proposed method to cases where the macroscale localization pattern is not known a-priori. Here, Alt. III is used to reduce the computational cost.

\subsection{Elastic specimen with vertical localization band}

To investigate the SVE size dependence for different approximations to Eq. (21), we consider the specimen shown in Fig. 5. The specimen has length $L=10 \mathrm{~mm}$, height $H=5 \mathrm{~mm}$, notch diameter $D=0.5 \mathrm{~mm}$ and a thickness of $1 \mathrm{~mm}$. The bulk material is isotropic and linear elastic, with Young's modulus $E=210 \times 10^{3} \mathrm{MPa}$ and Poisson's ratio $v=0.3$. Plane strain is assumed. The interface is modeled by an isotropic elastic cohesive zone model with stiffness $k=1.0 \times 10^{5} \mathrm{~N} \mathrm{~mm}^{-3}$.

The left edge of the specimen is clamped and the right edge is subjected to a prescribed displacement according to $u_{x}=0.01 y / H, u_{y}=0$, see Fig. 6 .

The response of the specimen is computed in two ways: i) with DNS and ii) with $\mathrm{FE}^{2}$ for the interface response. For the DNS, we use a standard discretization with 6-node triangles in the bulk and model the interface by means of XFEM using the previously mentioned isotropic elastic interface law. ${ }^{8}$ For the $\mathrm{FE}^{2}$, we also use 6-node triangles with isotropic linear elastic material model (i.e. no homogenization of bulk response in this example) and an XFEM discretization of the interface. However, the interface response is now computed from SVEs in the cohesive zone Gauss points.

As output from the simulations, we choose to monitor the normal traction along the cohesive interface (Fig. 7). The normal tractions predicted with Alt. I and Alt. II are shown in Fig. 7a, b, respectively. As can be seen, these approximations both agree very well with the DNS solution. Alt. III, shown in Fig. 7c, agrees very well with the DNS for the smallest SVE, but large discrepancies occur for the largest SVE. This is expected, because Alt. III neglects the bulk strain in the SVE, and the bulk strain is more important for large SVEs.

In summary, all three approximations are accurate for the smallest SVE (i.e. when strong scale separation holds), whereas Alt. I and Alt. II perform better than Alt. III when the SVE size approaches the size of the macroelements.

\footnotetext{
${ }^{8}$ For this example, the discretization for the DNS is straightforward (and cheaper than the $\mathrm{FE}^{2}$ in terms of computational cost). Therefore, there is no benefit of using $\mathrm{FE}^{2}$ for this example from a computational point of view. However, the example is interesting because it demonstrates the SVE size dependence of the different approximations.
} 


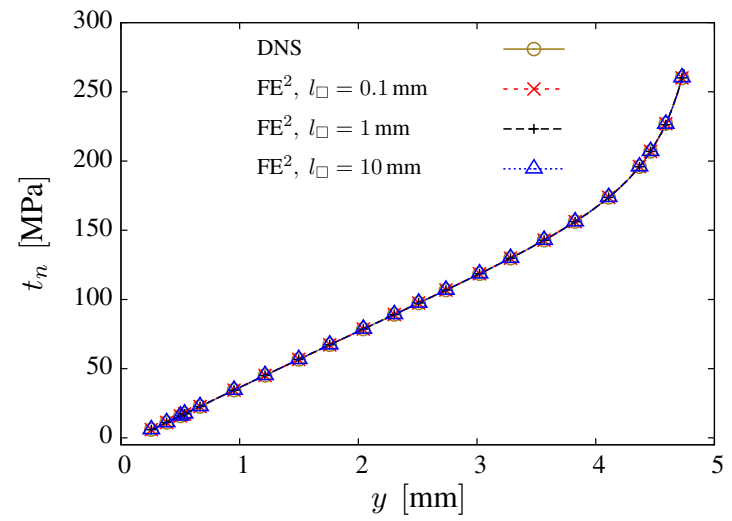

(a)

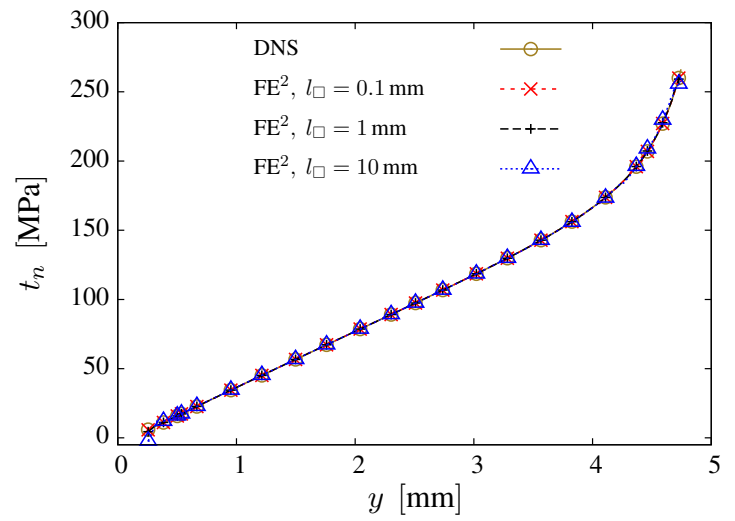

(b)

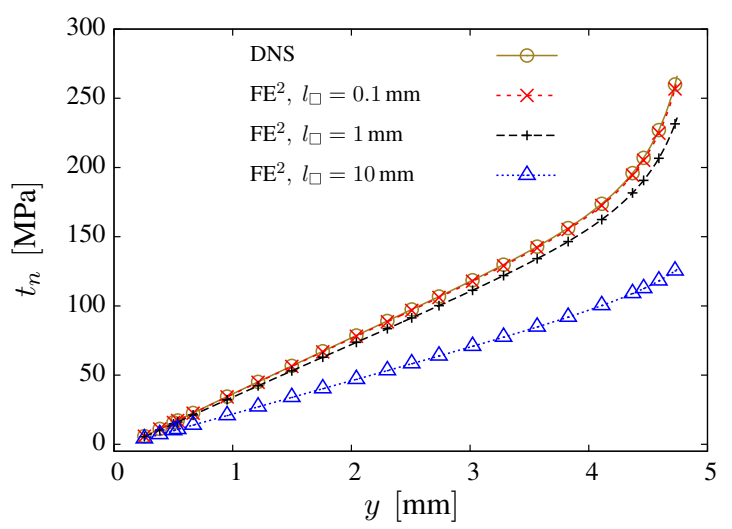

(c)

Fig. 7 Normal traction along the interface for Example 4.2. a Alt. I, b Alt. II, c Alt. III

\subsection{Plate with inclined effective discontinuity surface}

To investigate how the orientation of the effective discontinuity surface influences the macroscopic response, we consider a square plate with a circular hole and a band of porous material as shown in Fig. 8. The orientation of this softer band is described by the angle $\alpha$ between the band and the x-axis.

We consider an isotropic and linear elastic bulk material in plane strain with Young's modulus $E=210 \times 10^{3} \mathrm{MPa}$ and Poisson's ratio $v=0.3$. The material is homogeneous, except in the softer band, where small holes in the material are present as shown in Figs. 9 and 10. The side length of the plate is $L=100 \mathrm{~mm}$ and the diameter of the macroscopic hole is $d=60 \mathrm{~mm}$. The small holes in the soft band have a diameter of $1 \mathrm{~mm}$, and the spacing between the small holes (centercenter distance) is $1.25 \mathrm{~mm}$. Three rows of small holes run across the entire plate as shown in Figs. 9 and 10.

We solve the problem using both DNS and $\mathrm{FE}^{2}$ for different values of the angle $\alpha$. DNS meshes for three different angles are shown in Fig. 9. For the DNS meshes, 3-node triangles with a side length of $1 \mathrm{~mm}$ are used, except in the region surrounding the small holes, where a side length of $0.025 \mathrm{~mm}$ is used. For the $\mathrm{FE}^{2}$ simulations, 6-node triangles with an element side length of $5.0 \mathrm{~mm}$ is used on the macroscale. For the SVE meshes, 6-node triangles with a side length of $0.5 \mathrm{~mm}$ are used, except in the region surrounding the small holes, where a side length of $0.1 \mathrm{~mm}$ is used. Regarding the SVE size, we choose $l_{\square}=20 \mathrm{~mm}$.

The left edge of the specimen is clamped, and a uniform displacement of $u_{0}=0.01 \mathrm{~mm}$ is applied in the $\mathrm{x}$-direction on the right edge. As output from the simulations, we choose to monitor the reaction force in the $\mathrm{x}$-direction.

The deformed shape of the structure, computed with DNS for $\alpha=60^{\circ}$, is shown in Fig. 11. As can be seen, a substantial amount of the deformation is localized to the soft band. This is also reflected in the stress field shown in Fig. 12: the effective stress is much higher around the holes than in the rest of the structure.

The reaction force on the clamped edge, computed with both DNS and $\mathrm{FE}^{2}$, is shown for different $\alpha$ in Fig. 13. The agreement between the DNS and $\mathrm{FE}^{2}$ simulations is good, the largest difference in reaction force between the DNS and the $\mathrm{FE}^{2}$ simulations is $7 \%$ (for $\alpha=45^{\circ}$ ). The larger discrepancy close to $\alpha=45^{\circ}$ is most likely due to the finite width of the 


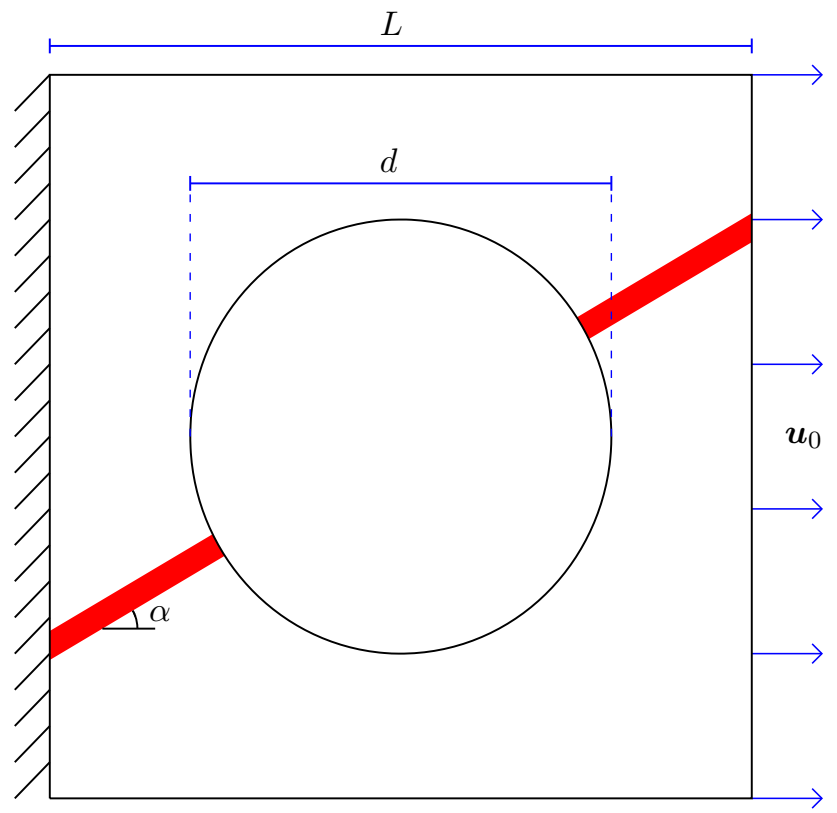

Fig. 8 Macroscale geometry considered in Example 4.3: a square plate with hole. The plate is clamped at the left side and a uniform displacement is imposed on the right side. The thick red stripe indicates the location of the effective discontinuity surface $\bar{\Gamma}_{d}$. (Color figure online)

soft region. More precisely, the DNS will capture the effect of the finite width soft region overlapping the upper right corner, whereas the $\mathrm{FE}^{2}$ macroscale representation of the soft region is a strong discontinuity with zero (macroscale) thickness. Despite the discrepancy around $\alpha=45^{\circ}$, we conclude that the agreement is good for all values of $\alpha$.

\subsection{Nonlinear response of a plate with a softening region}

To verify that the response during softening is insensitive to the SVE size, we consider a plate with a softening region containing a cohesive surface and holes as shown in Fig. 14. The plate has side length $L=100 \mathrm{~mm}$ and thickness $1 \mathrm{~mm}$ (plane strain is assumed). The cohesive band has holes with radius $1 \mathrm{~mm}$ and center-center distance $4 \mathrm{~mm}$, and runs through the center of the plate at an angle of $\alpha=25^{\circ}$ to the horizontal axis.

The bulk material is linear elastic with Young's modulus $E=20.0 \times 10^{3} \mathrm{MPa}$ and Poisson's ratio $v=0.2$.

The cohesive interface is modeled by a standard bilinear cohesive law. In this example, we consider monotonic loading and thus disregard history effects in the cohesive zone material, thereby employing an "elastic" cohesive law with softening as indicated in Fig. 15. For the cohesive zone material parameters, we choose the initial stiffness as $k=1.0 \times 10^{5} \mathrm{~N} \mathrm{~mm}^{-3}$, the peak stress as $\sigma_{f n}=$ $2.5 \times 10^{2} \mathrm{MPa}$ and the fracture energy as $G_{I}=2.0 \times$ $10^{1} \mathrm{~N} \mathrm{~mm}^{-3}$.

For the DNS, the bulk material is discretized with 6-node triangles with a side length of $1 \mathrm{~mm}$, except close to the interface, where the side length is $0.075 \mathrm{~mm}$. The cohesive surface is modeled by interface elements. For the $\mathrm{FE}^{2}$ simulations, $\mathrm{XFEM}$ is used on the macroscale, whereas interface elements are used to represent the microscale discontinuity. Regarding the bulk discretization, 6-node triangles with a side length of $1.25 \mathrm{~mm}$ are used on the macroscale. For the microscale, we use 6-node triangles with a side length of $0.36 \mathrm{~mm}$, except close to the interface where the side length is reduced to $0.18 \mathrm{~mm}$. Two SVE sizes are considered: $l_{\square}=3.62 \mathrm{~mm}$ and $l_{\square}=7.24 \mathrm{~mm}$, cf. Fig. 16 .

A prescribed displacement $\boldsymbol{u}_{0}$ as indicated in Fig. 14 is added incrementally in 10 load steps. As output from the simulations, we choose to monitor the reaction force for the lower right support. The reaction force vs displacement curve for the lower right support, computed with DNS and $\mathrm{FE}^{2}$, is shown in Fig. 17. As can be seen, the $\mathrm{FE}^{2}$ solution is insensitive to the SVE size and agrees very well with the DNS solution.

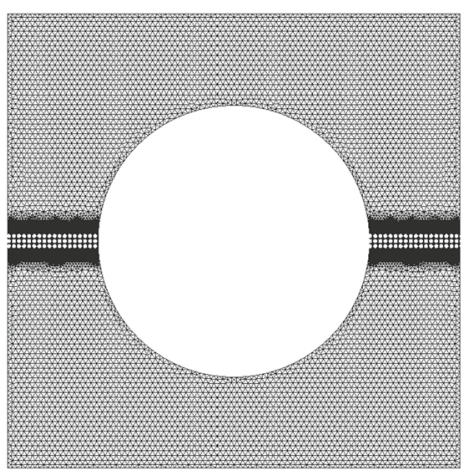

(a)

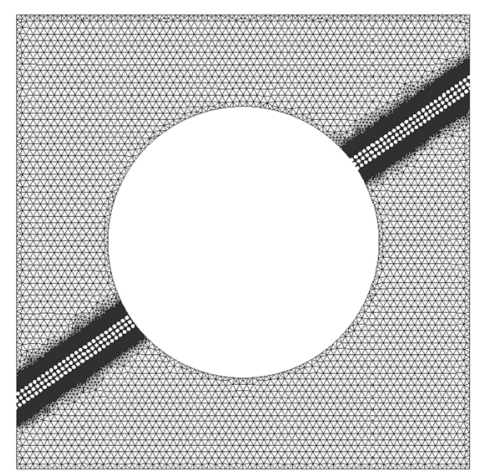

(b)

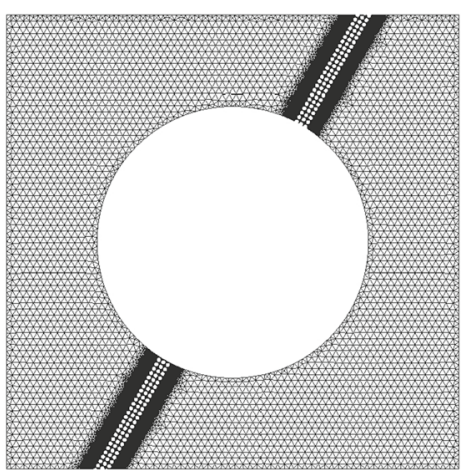

(c)

Fig. 9 Meshes for fully resolved simulations (DNS) of the plate considered in Example 4.3. Meshes for three different orientations of the soft band are shown. $\mathbf{a} \alpha=0^{\circ}, \mathbf{b} \alpha=35^{\circ}, \mathbf{c} \alpha=60^{\circ}$ 
Fig. 10 Meshes for two-scale simulation of the plate considered in Example 4.3: macroscale mesh (left) and SVE mesh (right) for $\alpha=35^{\circ}$

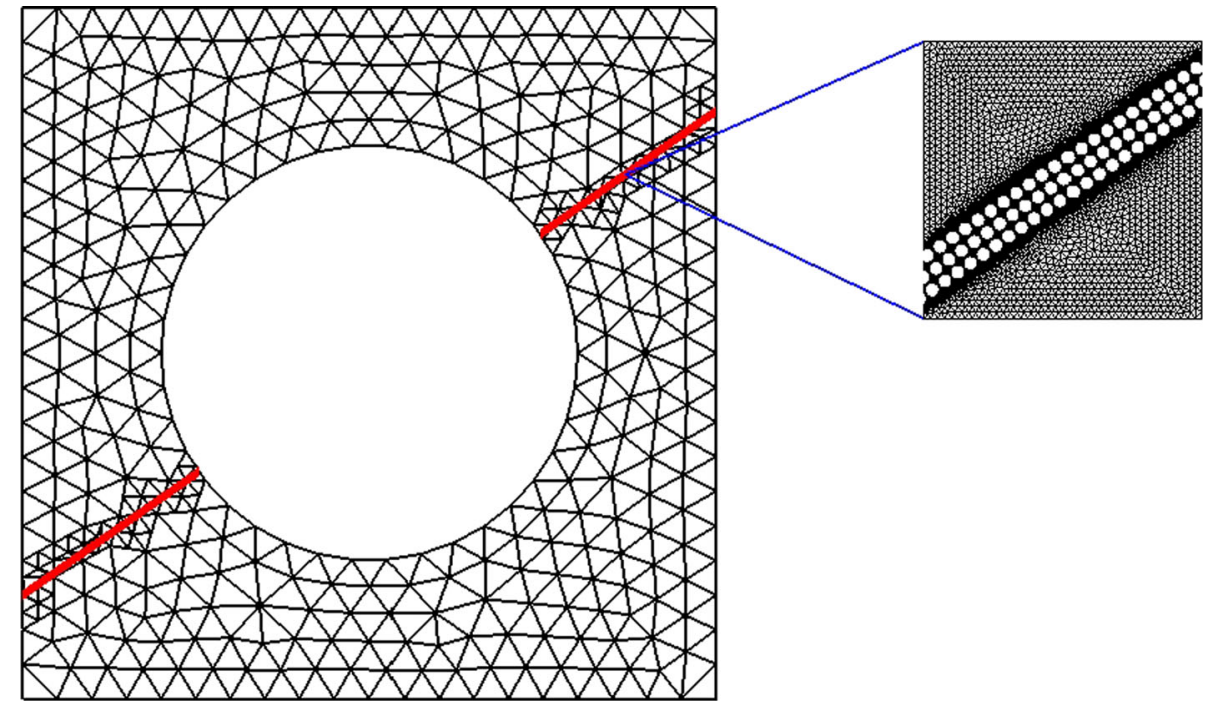

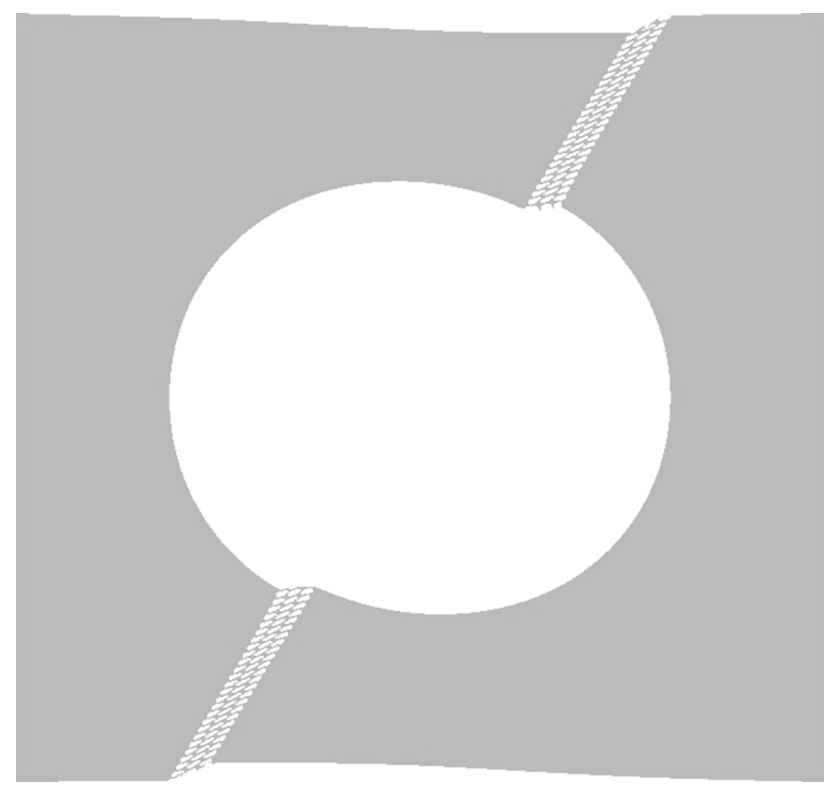

Fig. 11 Fully resolved simulation (DNS) of the plate considered in Example 4.3. The figure shows the deformed shape of the plate magnified by a factor of 500 for $\alpha=60^{\circ}$

\subsection{Plate with localizing microcracks}

In this example, we consider a plate with a circular hole and a microstructure consisting of a matrix with circular inclusions as shown in Fig. 18. The plate, which has length $L=50 \mathrm{~mm}$ and hole diameter $D=20 \mathrm{~mm}$, is clamped at the left edge. The right edge is fixed in the horizontal direction and a uniform displacement is applied in the vertical direction.

The matrix material is isotropic and linear elastic with Young's modulus $E_{m}=210 \times 10^{3} \mathrm{MPa}$ and Poisson's ratio $v_{m}=0.3$. The inclusions are also isotropic and linear elastic, with Young's modulus $E_{i}=10.0 \times 10^{3} \mathrm{MPa}$ and Poisson's ratio $v_{i}=0.3$. The inclusions have diameter $d=0.5 \mathrm{~mm}$ and are randomly distributed in the plate. For the microscale problem in the $\mathrm{FE}^{2}$ simulations and the DNS, cracks nucleate if the highest principal stress exceeds $400 \mathrm{MPa}$. Crack propagation is modeled using the concept of material forces, whereby the cracks propagate in the direction of the material force when the magnitude of the material force exceeds $0.25 \mathrm{~N}$. On the macroscale, we consider two different meshes as shown in Fig. 19. For the macroscale crack initiation, we choose for simplicity ${ }^{9}$ to nucleate cracks when the highest principal strain exceeds $1 \times 10^{3}$ in any integration point. A macroscopic crack segment is thereby introduced in the direction perpendicular to the corresponding principal strain direction. Such macroscopic new segment is also allowed to intersect already existing cracks on the macroscale. Existing macroscale cracks propagate in the direction perpendicular to the highest principal strain when the highest principal strain in the integration point closest to the crack tip exceeds $1 \times 10^{-4}$.

The resulting effective stress predicted with DNS is shown in Fig. 20, and the displacement field, computed with DNS and $\mathrm{FE}^{2}$, is shown in Fig. 21. In the DNS, the propagating microcracks interact with the microstructure to form an irregularly shaped macrocrack. The $\mathrm{FE}^{2}$ simulation does not capture the small scale irregularities in the crack pattern on the macroscale, but the overall crack pattern is in qualitative agreement with the DNS. The reaction forces computed with DNS and $\mathrm{FE}^{2}$ are shown in Fig. 22. Despite the crude macroscale crack initiation criterion adopted in the present example, the $\mathrm{FE}^{2}$ solution agrees fairly well with the DNS.

\footnotetext{
9 A more rigorous approach would be to detect singularity of the acoustic tensor. However, a simple strain based criterion is computationally convenient for the example considered here.
} 


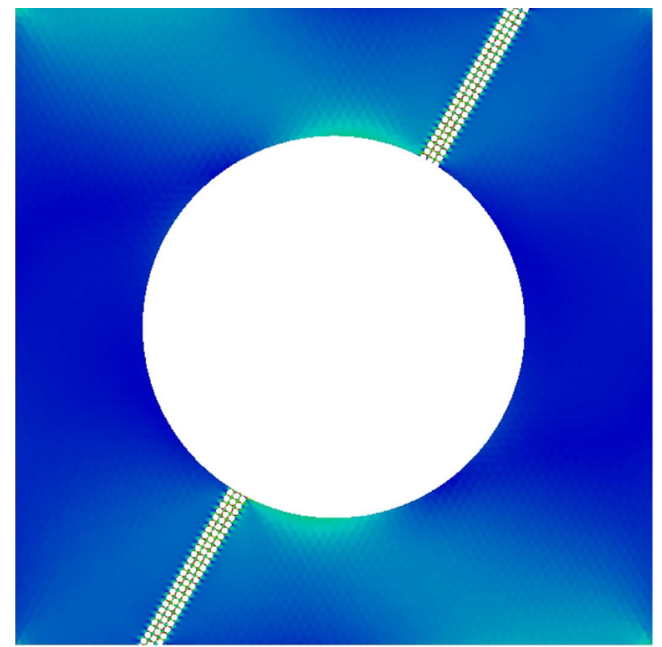

(a)

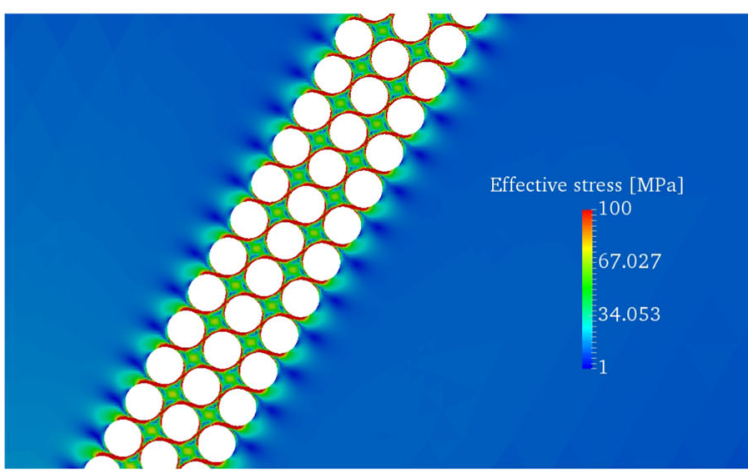

(b)

Fig. 12 Fully resolved simulation (DNS) of the plate considered in Example 4.3. The figure shows the stress distribution (limited to $100 \mathrm{~mm}$ ). a Overall stress distribution. b Stress distribution in the porous band

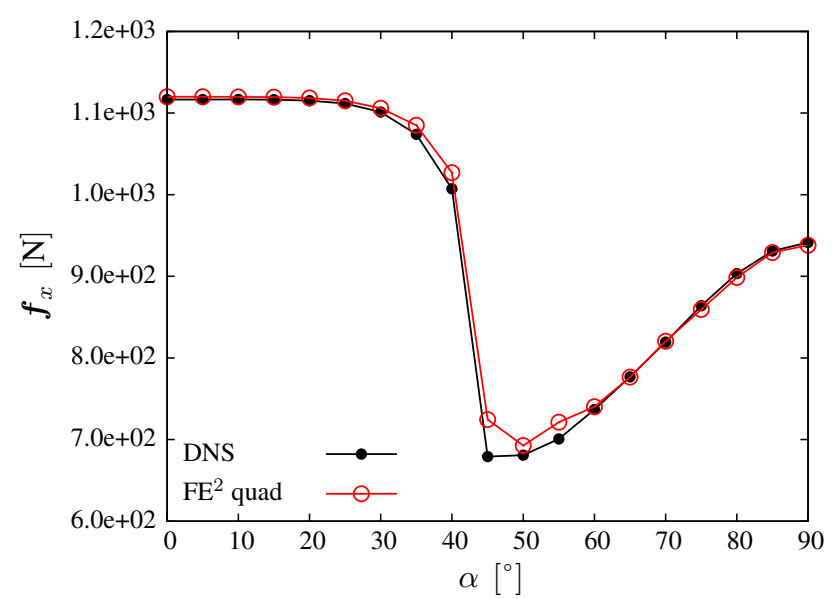

Fig. 13 Plate with soft band considered in Example 4.3. Horizontal reaction force versus angle of the soft band computed with DNS and $\mathrm{FE}^{2}$

\section{Conclusions}

In the present work, we model fracturing solids undergoing strain localization using a two-scale approach based on Variationally Consistent Homogenization (VCH). We propose a continuous-discontinuous homogenization scheme, where the eXtended Finite Element Method (XFEM) is used to represent narrow macroscale localization bands. The displacement discontinuities across the macroscopic discontinuity surfaces are transferred to the microscale in a smeared sense, using a smearing width $l_{d}$ that is related to the orientation of the effective discontinuity and the SVE size. Using a correct smearing width turns out to be crucial for obtaining accurate results without pathological dependence on the SVE size.

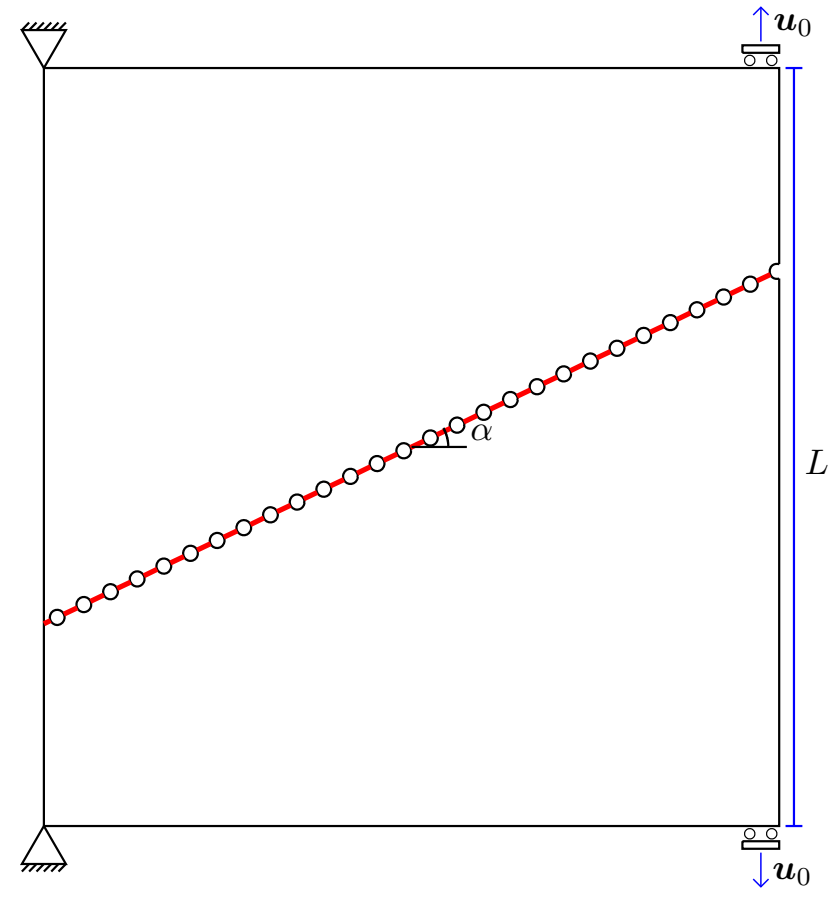

Fig. 14 Plate containing a softening region with a cohesive surface (red) and holes. (Example 4.4). (Color figure online)

Adopting the procedure of $\mathrm{VCH}$ and assuming that the macroscale localization region is well approximated by a strong discontinuity, we derive a two-scale scheme containing a conventional bulk term, a term of cohesive zone type and a correction term. The latter term has negligible effect on the accuracy if strong scale separation holds, but is indeed needed to retain any (possible) symmetry of the problem. A major benefit of the proposed scheme is that it does not 


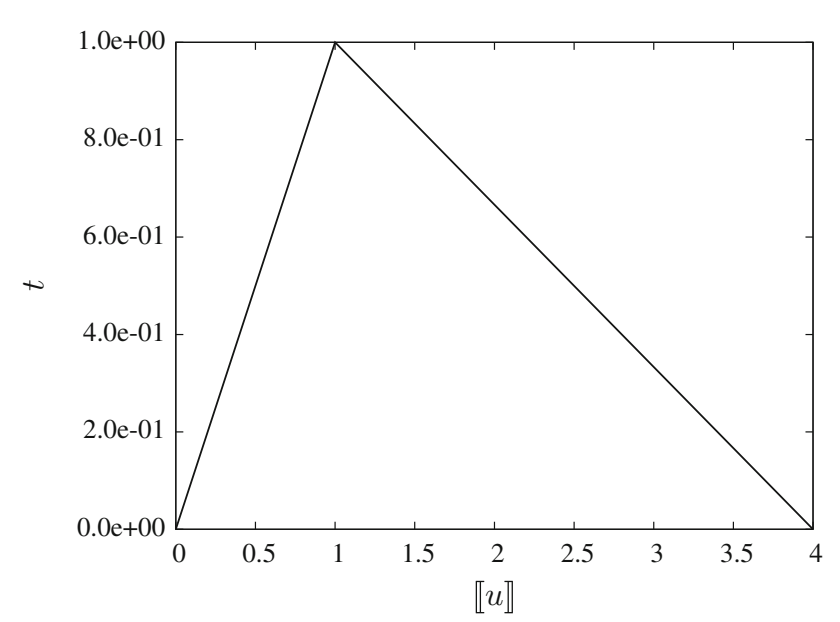

Fig. 15 Schematic illustration of the cohesive zone model adopted in Example 4.4

require dynamic tracking of an evolving damage region on the microscale, and that it does not explicitly assume a particular constitutive behavior on the microscale.

In the numerical examples, we show that the proposed scheme is insensitive to the SVE size if all terms obtained in the derivation are included, and that approximations are possible for sufficiently small SVE sizes $l_{\square}$ (i.e. in the case of strong scale separation). Furthermore, it is demonstrated that the proposed scheme, in combination with localization aligned weakly periodic BCs, is accurate for varying crack orientations. We also illustrate the potential of the proposed scheme with an $\mathrm{FE}^{2}$ simulation of a localizing microstructure. In the last example presented, the potential of the proposed method has been demonstrated using sim-

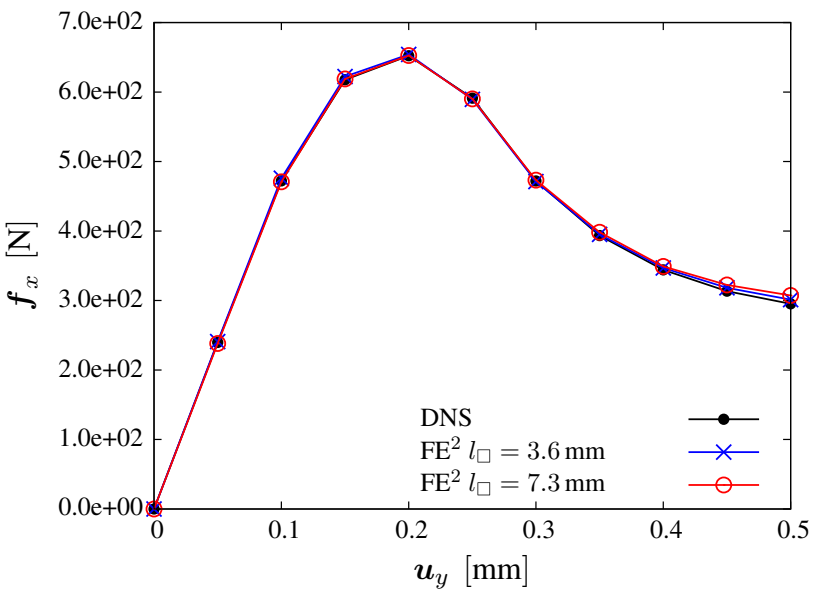

Fig. 17 Reaction force versus displacement for the lower right support (Example 4.4)

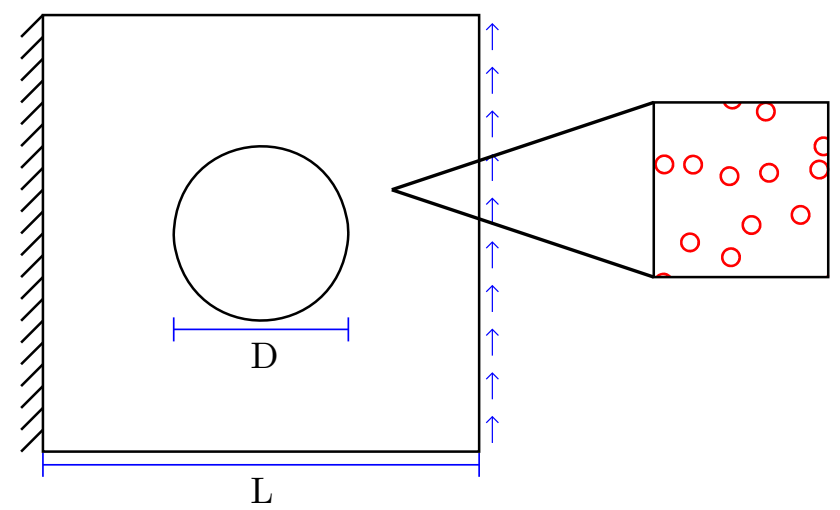

Fig. 18 Plate with hole considered in Example 4.5 (left). The plate has a microstructure consisting of a stiff matrix with soft circular inclusions, illustrated as red circles (right)
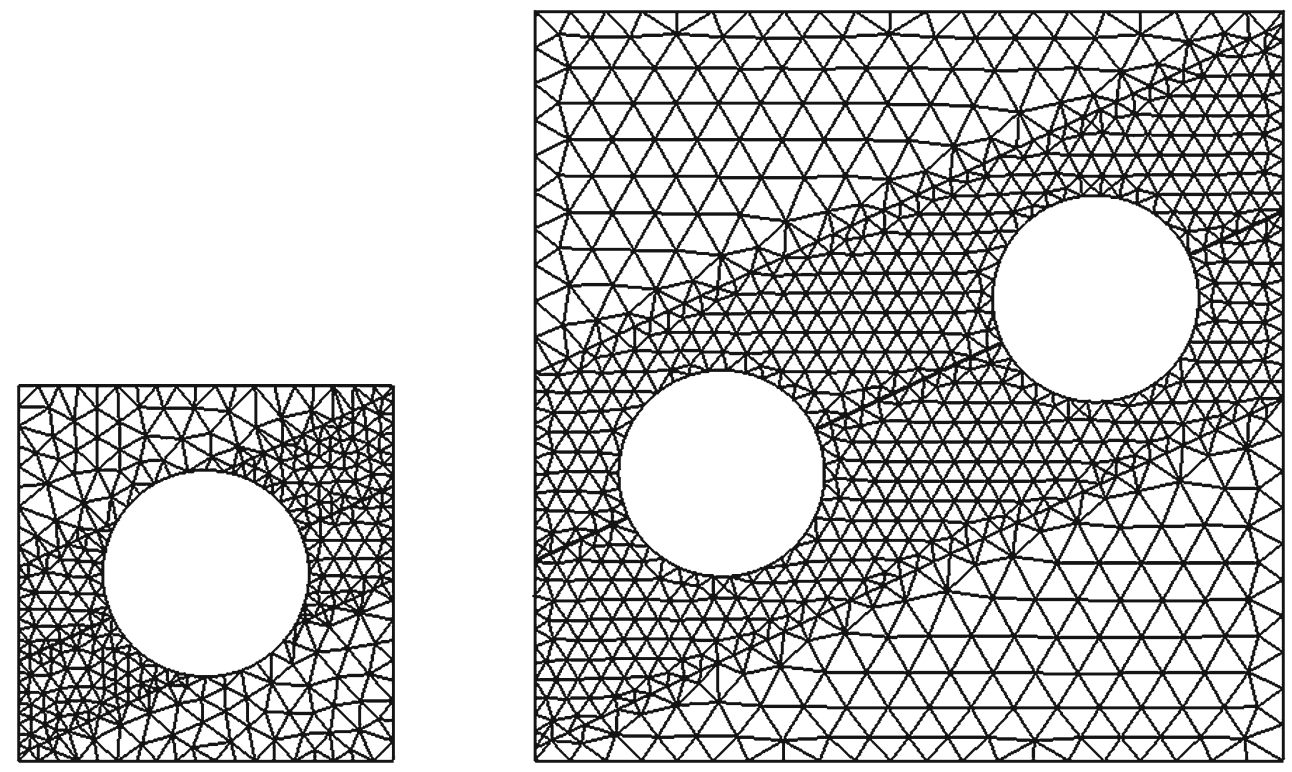

Fig. 16 SVEs considered in Example 4.4. The SVE sizes are $l_{\square}=3.62 \mathrm{~mm}$ (left) and $l_{\square}=7.24 \mathrm{~mm}$ (right) 


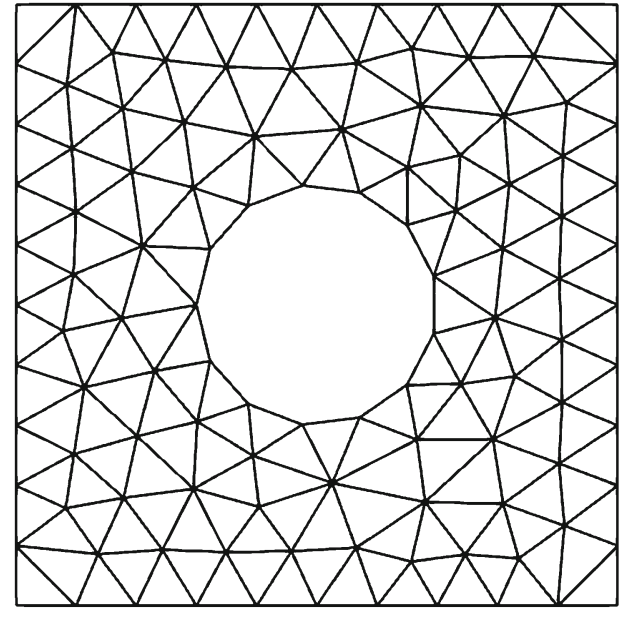

(a)

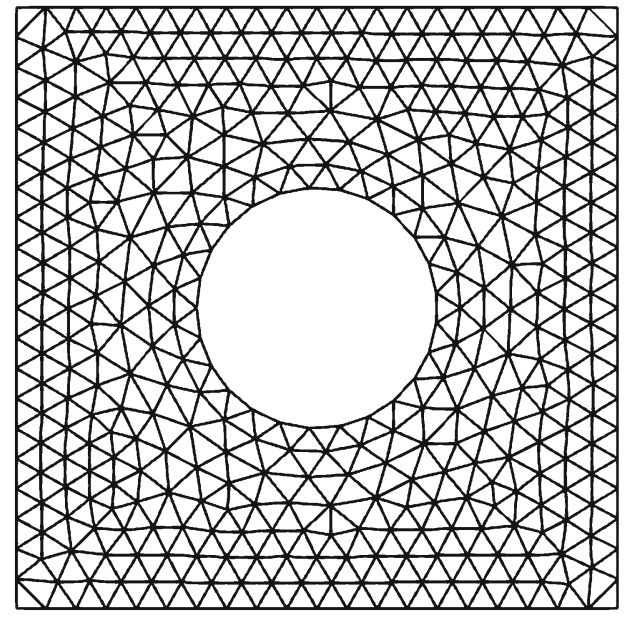

(b)

Fig. 19 Macro meshes considered in Example 4.5. a Mesh 1: 177 elements. b Mesh 2: 735 elements

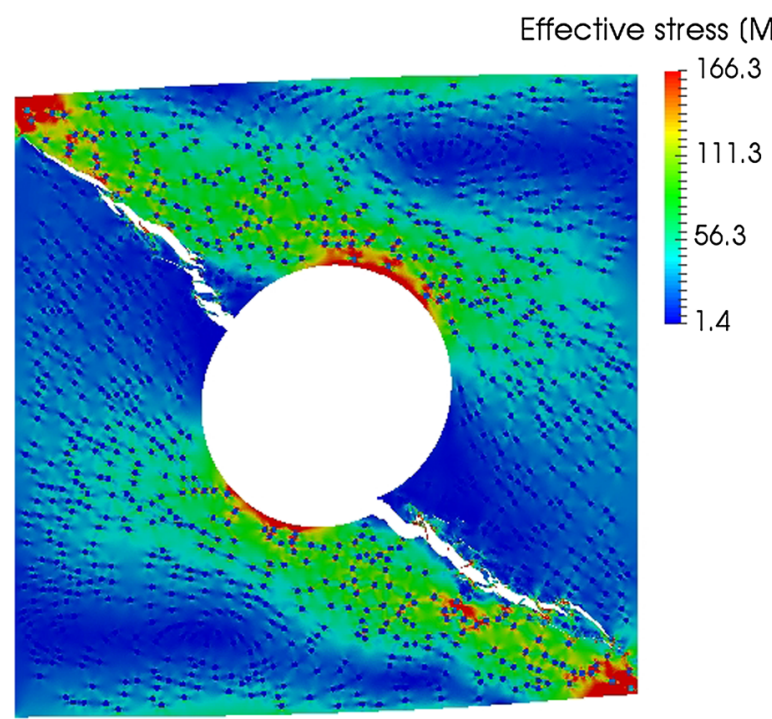

Fig. 20 Effective stress computed with DNS (Example 4.5)
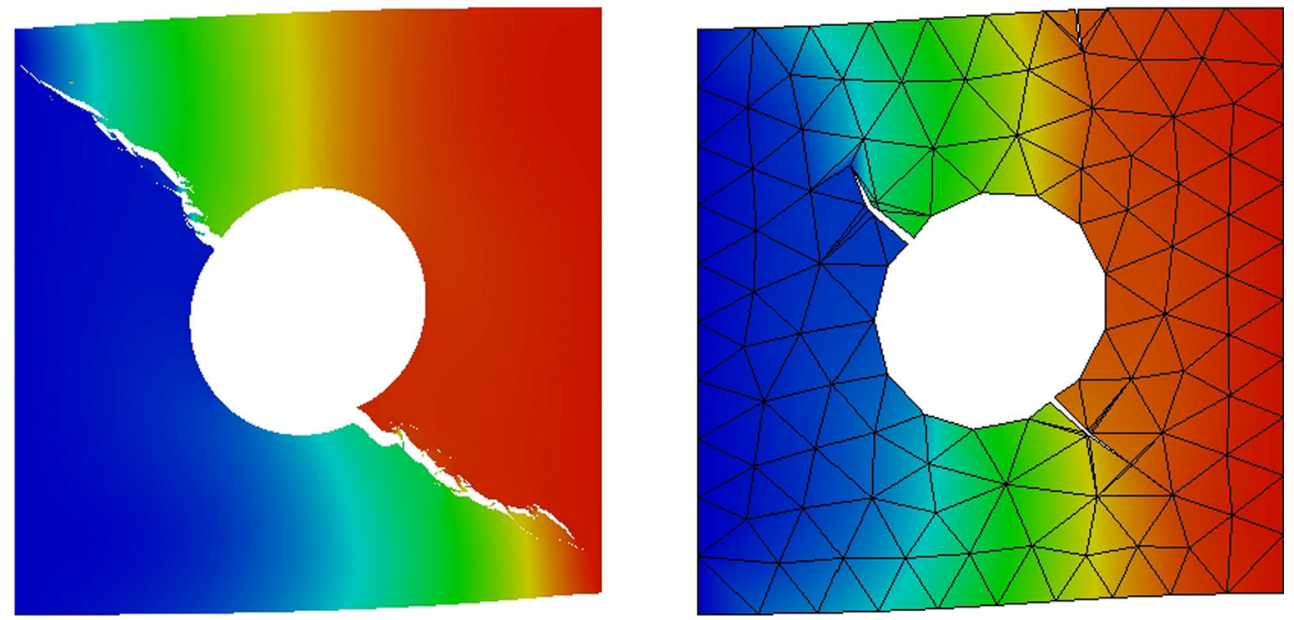

Fig. 21 Displacement field computed with DNS (left) and $\mathrm{FE}^{2}$ (right) for Example 4.5

Fig. 22 Reaction force versus displacement for a plate with coalescing

microcracks (Example 4.5)

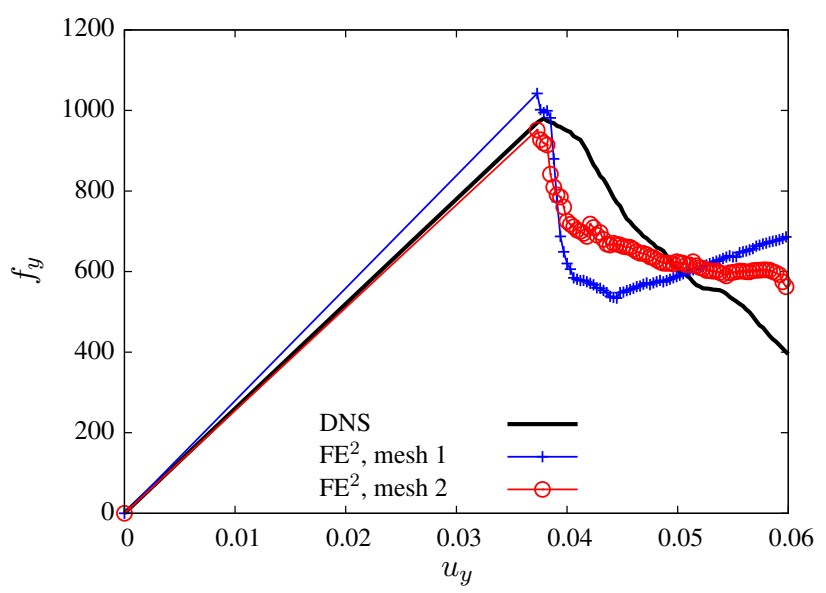


plified models for crack initiation and propagation. Future work therefore involves more accurate models for macroscale crack propagation, i.e. a proper strategy for insertion and propagation of (possible) macroscale discontinuities.

In summary, a multiscale modeling scheme that allows microscopic and macroscopic localization is proposed. The good performance of the proposed scheme is demonstrated by several numerical examples.

Acknowledgements The project is financially supported by the Swedish Research Council (www.vr.se) under contract 2012-3006. The simulations were performed on resources at Chalmers Centre for Computational Science and Engineering (C3SE) provided by the Swedish National Infrastructure for Computing (SNIC).

Open Access This article is distributed under the terms of the Creative Commons Attribution 4.0 International License (http://creativecomm ons.org/licenses/by/4.0/), which permits unrestricted use, distribution, and reproduction in any medium, provided you give appropriate credit to the original author(s) and the source, provide a link to the Creative Commons license, and indicate if changes were made.

\section{References}

1. Geers MGD, Kouznetsova VG, Brekelmans WAM (2010) Multiscale computational homogenization: trends and challenges. J Comput Appl Math 234(7):2175-2182

2. Nguyen VP, Stroeven M, Sluys LJ (2011) Multiscale continuous and discontinuous modeling of heterogeneous materials: a review on recent developments. J Multiscale Model 3(04):229-270

3. Coenen EWC, Kouznetsova VG, Geers MGD (2012) Novel boundary conditions for strain localization analyses in microstructural volume elements. Int J Numer Methods Eng 90(1):1-21

4. Ostoja-Starzewski M (2006) Material spatial randomness: from statistical to representative volume element. Probab Eng Mech 21(2):112-132

5. Verhoosel CV, Remmers JJC, Gutiéerrez MA, de Borst R (2010) Computational homogenization for adhesive and cohesive failure in quasi-brittle solids. Int J Numer Methods Eng 83(8-9):11551179

6. Coenen EWC, Kouznetsova VG, Bosco E, Geers MGD (2012) A multi-scale approach to bridge microscale damage and macroscale failure: a nested computational homogenizationlocalization framework. Int J Fract 178(1-2):157-178

7. Souza FV, Allen DH (2011) Modeling the transition of microcracks into macrocracks in heterogeneous viscoelastic media using a twoway coupled multiscale model. Int J Solids Struct 48(22-23):31603175
8. Belytschko T, Loehnert S, Song JH (2008) Multiscale aggregating discontinuities: a method for circumventing loss of material stability. Int J Numer Methods Eng 73(6):869-894

9. Bosco E, Kouznetsova VG, Geers MGD (2015) Multi-scale computational homogenization-localization for propagating discontinuities using X-FEM. Int J Numer Methods Eng 102(3-4):496-527 arXiv: 1010.1724

10. Toro S, Sánches PJ, Blanco PJ, De Souza Neto EA, Huespe AE, Feijóo RA (2016) Multiscale formulation for material failure accounting for cohesive cracks at the macro and micro scales. Int J Plast 76:75-110

11. Nguyen VP, Lloberas Valls O, Stroeven M, Sluys LJ (2010) On the existence of representative volumes for softening quasi-brittle materials: a failure zone averaging scheme. Comput Methods Appl Mech Eng 199(45-48):3028-3038

12. Svenning E, Fagerström M, Larsson F (2016) On computational homogenization of microscale crack propagation. Int $\mathrm{J}$ Numer Methods Eng 108:76-90

13. Svenning E, Fagerström M, Larsson F (2016) Localization aligned weakly periodic boundary conditions. Int J Numer Methods Eng (2016). doi:10.1002/nme.5483

14. Larsson F, Runesson K (2008) Adaptive bridging of scales in continuum modeling based on error control. Int J Multiscale Comput Eng 6(4):371-392

15. Larsson F, Runesson K, Su F (2010) Variationally consistent computational homogenization of transient heat ow. Int J Numer Methods Eng 81(13):1659-1686

16. Hughes TJR, Feijóo GR, Mazzei L, Quincy JB (1998) The variational multiscale method: a paradigm for computational mechanics. Comput Methods Appl Mech Eng 166(1-2):3-24

17. Belytschko T, Black $T$ (1999) Elastic crack growth in finite elements with minimal remeshing. Int $\mathrm{J}$ Numer Methods Eng 45(5):601-620

18. Babuska I, Caloz G, Osborn JE (1994) Special finite element methods for a class of second order elliptic problems with rough coefficients. SIAM J Numer Anal 31(4):945-981

19. Fries TP, Belytschko T (2010) The extended/generalized finite element method: an overview of the method and its applications. Int J Numer Methods Eng 84(3):253-304

20. Larsson F, Runesson K, Saroukhani S, Vafadari R (2011) Computational homogenization based on a weak format of micro-periodicity for RVE-problems. Comput Methods Appl Mech Eng 200(1-4):1126

21. Svenning E, Fagerström M, Larsson F (2016) Computational homogenization of microfractured continua using weakly periodic boundary conditions. Comput Methods Appl Mech Eng 299:1-21

22. Patzák B, Bittnar Z (2001) Design of object oriented finite element code. Adv Eng Softw 32(10-11):759-767

23. Patzák B (2000) OOFEM project home page, www.oofem.org 